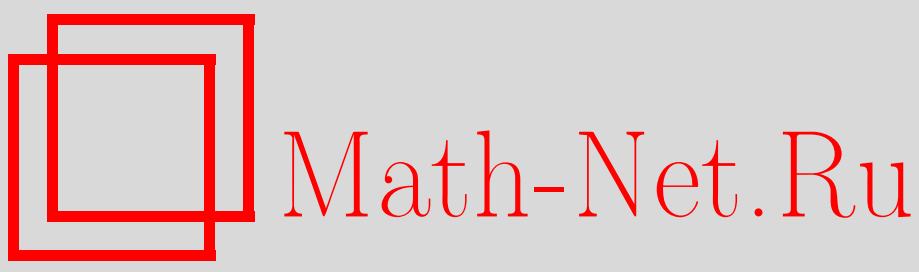

М. И. Вишик, В. В. Чепыжов, Колмогоровская $\varepsilon$-энтропия аттракторов систем реакции-диффузии, Матем. сб., 1998, том 189, номер 2, 81-110

DOI: https://doi.org/10.4213/sm301

Использование Общероссийского математического портала Math-Net.Ru подразумевает, что вы прочитали и согласны с пользовательским соглашением

http://www . mathnet.ru/rus/agreement

Параметры загрузки:

IP : 34.227 .88 .159

26 апреля 2023 г., 13:02:24 
УДК 517.95

\author{
М.И. Вишик, В.В. Чепыжов
}

\title{
Колмогоровская $\varepsilon$-энтропия аттракторов систем реакции-диффузии
}

\begin{abstract}
Изучается колмогоровская $\varepsilon$-энтропия равномерного аттрактора $\mathscr{A}$ семейства неавтономных уравнений реакции-диффузии с внешней силой $g(x, t)$. Предполагается, что $g(x, t)$ принадлежат трансляционно-инвариантному относительно групшы сдвигов по $t$ множеству $\Sigma, \Sigma \subset C(\mathbb{R} ; H), H=\left(L_{2}(\Omega)\right)^{N}$. Кроме того, $\Sigma$ компактно в $C(\mathbb{R} ; H)$.

В работе дается оценка $\varepsilon$-энтропии равномерного аттрактора $\mathscr{A}$ через $\varepsilon_{1}=\varepsilon_{1}(\varepsilon)$-энтропию компактного в $C([0, l] ; H)$ множества $\Sigma_{l}$ внешних сил $g(x, t) \in \Sigma$, суженных на интервал $[0, l], l=l(\varepsilon)\left(\varepsilon_{1}(\varepsilon) \sim \mu \varepsilon, l(\varepsilon) \sim \tau \log _{2}(1 / \varepsilon)\right)$. Эта общая оценка иллюстрируется рядом примеров, взятых из различных областей математической физики и теории информации.

Библиографйл: 23 названия.
\end{abstract}

В статье исследуется система реакции-диффузии с зависящей от времени внешней силой $g(x, t)$. При изучении таких неавтономных систем естественно рассматривать семейство систем с внешними силами, принадлежащими некоторому трансляционно-инвариантному множеству $\Sigma$, которое содержит начальную внешнюю силу $g_{0}(x, t)$. Таким образом, рассматривается семейство уравнений

$$
\begin{gathered}
\partial_{t} u=\nu \Delta u-f(u)+g(x, t), \quad t \in \mathbb{R}, \\
\left.u\right|_{\partial \Omega}=0, \quad x \in \Omega, \quad \Omega \Subset \mathbb{R}^{n},
\end{gathered}
$$

где $u=u(x, t)=\left(u^{1}, \ldots, u^{N}\right)$ является неизвестной вектор-функцией, $f=$ $\left(f^{1}, \ldots, f^{N}\right), g=\left(g^{1}, \ldots, g^{N}\right)$, а $\nu$ - положительный параметр. Функция $f$ удовлетворяет некоторым условиям, описанным в $\S 1$, которые обеспечивают однозначную разрешимость соответствуюшей задачи Коши. Внешние силы $g(x, s)=g(s), s \in \mathbb{R}$, принадлежат множеству $\Sigma, \Sigma \subset C(\mathbb{R} ; H), H=\left(L_{2}(\Omega)\right)^{N}$. Пространство $C(\mathbb{R} ; H)$ снабжено топологией равномерной сходимости на любом отрезке числовой оси $\mathbb{R}$, т.е., по определению, $g_{n}(s)$ сходится к $g(s)$ в $C(\mathbb{R} ; H)$ при $n \rightarrow \infty$, если

$$
\max _{s \in\left[t_{1}, t_{2}\right]}\left\|g_{n}(s)-g(s)\right\|_{H} \rightarrow 0, \quad n \rightarrow \infty,
$$

для любого $\left[t_{1}, t_{2}\right] \Subset \mathbb{R}$. Предполагается, что

(I) множество $\Sigma$ компактно в $C(\mathbb{R} ; H)$;

Работа вьполнена при частичной поддержке Российского фонда фундаментальных исследований (грант № 96-01-00354), а также the U.S. Civilian Research \& Development Foundation for the Independent States of the Former Soviet Union (CRDF) (грант № RM1-186). 
(II) множество $\Sigma$ трансляционно-инвариантно, т.е.

$$
T(h) \Sigma=\Sigma \quad \forall h \in \mathbb{R}
$$

где $T(h) g(s)=g(h+s),\{T(h): h \in \mathbb{R}\}(\{T(h)\}$ называется трансляционной әрyпnой).

Пусть

$$
\left.u\right|_{t=\tau}=u_{\tau}, \quad u_{\tau} \in H, \quad \tau \in \mathbb{R},
$$

является начальным условием для (0.1). Любое решение $u(t), t \geqslant \tau$, задачи $(0.1),(0.2)$ и (0.4) может быть представлено в виде:

$$
u(t)=U_{g}(t, \tau) u_{\tau}, \quad t \geqslant \tau, \quad \tau \in \mathbb{R}
$$

При заданном $g \in \Sigma$ двупараметрическое семейство операторов $\left\{U_{g}(t, \tau), t \geqslant \tau\right.$, $\tau \in \mathbb{R}\}, U_{g}(t, \tau): H \rightarrow H$, называется процессом, отвечающим задаче $(0.1),(0.2)$, $(0.4)$ с внешней силой $g(s) \in \Sigma$. Очевидно, $U_{g}(t, \tau)=U_{g}(t, \theta) U_{g}(\theta, \tau) \forall t \geqslant \theta \geqslant \tau$, $\tau \in \mathbb{R}$, и $U_{g}(\tau, \tau)=$ Id есть тождественньй оператор.

Компактное множество $\mathscr{A}_{\Sigma} \Subset H$ называется равномерным аттрактором семейства процессов $\left\{U_{g}(t, \tau)\right\}, g \in \Sigma$, если для каждого ограниченного множества $B \subset H$ и для любого $\tau \in \mathbb{R}$

$$
\sup _{g \in \Sigma} \operatorname{dist}_{H}\left(U_{g}(t, \tau) B, \mathscr{A}_{\Sigma}\right) \rightarrow 0, \quad t \rightarrow+\infty
$$

и при этом $\mathscr{A}_{\Sigma}$ является минимальньм компактным множеством, удовлетворяющим (0.6).

В работах [1] и [2] равномерный аттрактор $\mathscr{A}_{\Sigma}$ был построен в случае, когда $\Sigma=\mathscr{H}\left(g_{0}\right)$, а функция $g_{0}(s)$ является почти периодической со значениями в $H$. Здесь $\mathscr{H}\left(g_{0}\right)$ обозначает оболочку функции $g_{0}$. Класс почти периодических функций был существенно расширен в работах [3] и [4]. Теорема сушествования равномерного аттрактора была доказана для $\Sigma=\mathscr{H}\left(g_{0}\right)$, где $g_{0}(s)$ является транслящионно-компактной функцией в подходящем пространстве. Например, если $g_{0}$ трансляционно-компактна в $C(\mathbb{R} ; H)($ см. $\S 1)$, то условия $(\mathrm{I})$ и $(\mathrm{II})$ выполнены для $\Sigma=\mathscr{H}\left(g_{0}\right)$.

В статье [2] были получены верхние и нижние оценки для размерности равномерного аттрактора $\mathscr{A}_{\Sigma}$ в случае, когда $\Sigma=\mathscr{H}\left(g_{0}\right)$ является оболочкой квазипериодической функции $g_{0}(s)=\varphi\left(\alpha_{1} s, \ldots, \alpha_{k} s\right)$, где $\varphi\left(\omega_{1}, \ldots, \omega_{k}\right)$ - гладкая $2 \pi$-периодическая функция по каждому аргументу $\omega_{j}$ со значениями в $H$, а числа $\left(\alpha_{1}, \alpha_{2}, \ldots, \alpha_{k}\right)=\boldsymbol{\alpha}$ являются рационально независимыми (см. $\left.\S 1\right)$. Таким образом, в случае обшего положения пространство $\Sigma$ диффеоморфно $k$-мерному тору $\mathbb{T}^{k}$, и следовательно, $\operatorname{dim} \Sigma=k . \mathrm{B}[2]$ было доказано, что

$$
\operatorname{dim} \mathscr{A}_{\Sigma} \leqslant k+A_{1} k^{n /(n+2)}+A_{2}, \quad A_{1}, A_{2}=\text { const } .
$$

Значит, $\operatorname{dim} \mathscr{A}_{\Sigma}$ может быть оценено через $k=\operatorname{dim} \Sigma$. Кроме этого, были построены примеры показывающие, что

$$
\operatorname{dim} \mathscr{A}_{\Sigma} \geqslant k=\operatorname{dim} \Sigma .
$$


Следовательно, $\operatorname{dim} \mathscr{A}_{\Sigma}$ может стремиться к бесконечности при $k=\operatorname{dim} \Sigma \nearrow \infty$. Это явление побудило Фояша поставить задачу изучения колмогоровской $\varepsilon$-энтропии $\mathbf{H}_{\varepsilon}(\mathscr{A})$ (см. [5] и $\S 1$ ) равномерных аттракторов неавтономных эволюционных уравнений для общих трансляционно-компактных множеств $\Sigma$. Некоторые результаты в этом направлении были получены в работе [6] для двумерной системы Навье-Стокса с зависяшей от времени внешней силой.

В $\S 3$ изучается колмогоровская $\varepsilon$-энтропия $\mathbf{H}_{\varepsilon}\left(\mathscr{A}_{\Sigma}\right)=\mathbf{H}_{\varepsilon}\left(\mathscr{A}_{\Sigma}, H\right)$ равномерного аттрактора $\mathscr{A}_{\Sigma}$ в пространстве $H$ для системы уравнений реакции-диффузии $(0.1)$, когда $\Sigma$ удовлетворяет условиям (I) и (II). Установлена оценка сверху для $\mathbf{H}_{\varepsilon}\left(\mathscr{A}_{\Sigma}\right)$, которая зависит от $\mathbf{H}_{\varepsilon_{1}}(\Sigma, C([0, l] ; H))$ и от других параметров задачи. Здесь $\varepsilon_{1}=\varepsilon_{1}(\varepsilon), l=l(\varepsilon)$ (см. теорему 3.1).

Если фрактальная размерность $\mathbf{d}_{F}(\Sigma)<+\infty$, то нами доказана оценка для $\mathbf{d}_{F}\left(\mathscr{A}_{\Sigma}\right)$ через $\mathbf{d}_{F}(\Sigma)$. Если $\mathbf{d}_{F}(\Sigma)=+\infty$, приводится оценка $\mathbf{a d f}_{F}\left(\mathscr{A}_{\Sigma}\right)$ через $\mathbf{a d f}_{F}(\Sigma)$. Здесь $\mathbf{a d f} \mathbf{f}_{F}(B)$ обозначает функциональную размерность множества $B$ (см. [5]). Исследуется также зависимость метрического порядка $\mathbf{q}\left(\mathscr{A}_{\Sigma}\right)$ от метрического порядка $\mathbf{q}(\Sigma)$.

В $\S 2$ устанавливается свойство сжатия [7] для траекторий $u(t), v(t)$ системы $(0.1),(0.2)$, когда $u(t), v(t) \in \mathscr{A}_{\Sigma} \forall t \in \mathbb{R}$. Изучается зависимость от $t$ функций $\xi(t)=|u(t)-v(t)|$ и $\rho(t)=\|u(t)-v(t)\|^{2} /|u(t)-v(t)|^{2}$ (см. [7]), где $|\cdot|$ и $\|\cdot\|$ обозначают нормы в $H=\left(L_{2}(\Omega)\right)^{N}$ и в $H_{1}=\left(H_{0}^{1}(\Omega)\right)^{N}$, соответственно. Грубо говоря, доказано, что или $\left|Q_{m}(u(t)-v(t))\right| \leqslant\left|P_{m}(u(t)-v(t))\right|$, или $|u(t)-v(t)|$ мало, или $|u(t)-v(t)|$ экспоненциально убьвает. Здесь $P_{m}$ обозначает оператор ортогонального проектирования на линейное подпространство, натянутое на первые $m$ собственные функции оператора Лапласа $\Delta$ с нулевыми граничньми условиями, а $Q_{m}=I-P_{m}$. Свойство сжатия является основным инструментом при исследовании $\varepsilon$-энтропии в $\S 3$.

В $\S 4$ получены оценки $\varepsilon$-энтропии $\mathbf{H}_{\varepsilon}(\Sigma)$ некоторых специальных множеств $\Sigma$. Рассмотрены три типа таких множеств, взятых из различных областей математики. Из этих оценок, а также из теоремы 3.1 вытекают оценки для $\varepsilon$-энтропии $\mathbf{H}_{\varepsilon}\left(\mathscr{A}_{\Sigma}\right)$ равномерных аттракторов $\mathscr{A}_{\Sigma}$. Получены также оценки метрического порядка равномерных аттракторов $\mathscr{A}_{\Sigma}$ для всех рассмотренных типов множеств $\Sigma$.

\section{§1. Некоторые предварительные сведения}

Рассматривается семейство систем уравнений реакции-диффузии $(0.1),(0.2)$ с внешними силами $g(s)$, принадлежащими множеству $\Sigma \subset C(\mathbb{R} ; H)$, которое удовлетворяет условиям (I) и (II).

Вектор-функция $f(v) \in C^{1}\left(\mathbb{R}^{N} ; \mathbb{R}^{N}\right)$ удовлетворяет следующим условиям:

$$
\begin{gathered}
\gamma_{1}|v|^{p}-C_{1} \leqslant f(v) \cdot v \leqslant \gamma_{2}|v|^{p}+C_{2}, \quad \gamma_{i}>0, \quad p \geqslant 2, \\
\left|f_{v}(v)\right| \leqslant C_{3}\left(|v|^{p-2}+1\right), \quad-C_{4}|w|^{2} \leqslant f_{v}(v) w \cdot w \quad \forall v, w \in \mathbb{R}^{N} .
\end{gathered}
$$

Предполагается, что $p \leqslant 2(n-1) /(n-2)$ при $n \geqslant 3$ и $p$ - любое при $n \leqslant 2$. В этих предположениях задача $(0.1),(0.2),(0.4)$ имеет единственное решение $u \in$ $C\left(\mathbb{R}_{\tau} ; H\right) \cap L_{2}^{\text {loc }}\left(\mathbb{R}_{\tau} ; H_{1}\right)$ такое, что $\partial_{t} u \in L_{2}^{\text {loc }}\left(\mathbb{R}_{\tau} ; H_{-1}\right)$ (см. [2], [8], [9]). Здесь $\mathbb{R}_{\tau}=[\tau,+\infty)$ и $H_{-1}=H^{*}$ является пространством, сопряженным к $H_{1}$. Кроме 
того, $u \in C\left(\mathbb{R}_{\tau+\delta} ; H_{1}\right) \cap L_{2}^{\text {loc }}\left(\mathbb{R}_{\tau+\delta} ; H_{2}\right), H_{2}=\left(H^{2}(\Omega)\right)^{N}$, и $\partial_{t} u \in L_{2}^{\text {loc }}\left(\mathbb{R}_{\tau+\delta} ; H\right)$, где $\delta>0$ (см. [2], [8], [9]). (Условия (1.1), (1.2) могут быть обобшены.)

Таким образом, при заданном множестве $\Sigma$ задача $(0.1),(0.2),(0.4)$ порождает семейство процессов $\left\{U_{g}(t, \tau)\right\}, g \in \Sigma$, действующих в $H: U_{g}(t, \tau) u_{\tau}=u(t)$, где $u(t)$ есть решение $(0.1),(0.2),(0.4)$ с внешней силой $g \in \Sigma$.

Напомним некоторые определения, относящиеся к равномерным аттракторам семейств процессов, и сформулируем теорему о сушествовании и структуре аттракторов (см. также [1], [2], [10]-[15]).

Пусть задано семейство процессов $\left\{U_{g}(t, \tau)\right\}$, действуюших в банаховом пространстве $E$ и зависяших от параметра $g \in \Sigma$. Через $\mathscr{B}(E)$ обозначается семейство всех ограниченных множеств в $E$.

ОПРеДЕЛЕниЕ 1.1. Множество $P$ называется равномерно (относительно $g \in \Sigma)$ притягивающим для семейства процессов $\left\{U_{g}(t, \tau)\right\}, g \in \Sigma$, если при каждом $\tau \in \mathbb{R}$ и при любом $B \in \mathscr{B}(E)$

$$
\sup _{g \in \Sigma} \operatorname{dist}_{E}\left(U_{g}(t, \tau) B, P\right) \rightarrow 0, \quad t \rightarrow+\infty .
$$

Здесь, как обычно, $\operatorname{dist}_{E}(X, Y)=\sup _{x \in X} \operatorname{dist}_{E}(x, Y)=\sup _{x \in X} \inf _{y \in Y}\|x-y\|_{E}$.

ОПРЕДЕЛЕНИЕ 1.2. Компактноемножество $\mathscr{A}_{\Sigma} \Subset E$ называется равномерныцм (относительно $g \in \Sigma$ ) аттрактором семейства процессов $\left\{U_{g}(t, \tau)\right\}, g \in \Sigma$, если $\mathscr{A}_{\Sigma}$ является равномерно притягиваюшим множеством этого семейства и $\mathscr{A}_{\Sigma}$ принадлежит любому замкнутому равномерно притягиваюшему множеству (см. $[2],[15])$.

Легко показать, что если семейство процессов $\left\{U_{g}(t, \tau)\right\}, g \in \Sigma$, имеет компактное равномерно притягивающее множество $P \Subset E$, то сушествует и минимальное притягивающее множество, т.е. равномерный аттрактор $\mathscr{A}_{\Sigma} \subseteq P$.

Изучая семейство процессов, порожденное системой реакции-диффузии (0.1), $(0.2)$, легко установить, что существует равномерно притягивающее множество $B_{1}$, ограниченное в $H_{1}=\left(H_{0}^{1}(\Omega)\right)^{N}$ [2], [9], [16]. Следовательно, по теореме о компактности $B_{1}$ компактно в $H$ и, значит, существует равномерный аттрактор $\mathscr{A}_{\Sigma}$, ограниченный в $H_{1}$.

Для описания структуры множества $\mathscr{A}_{\Sigma}$ нам понадобятся некоторые дополнительные понятия.

ОПРЕДЕЛЕнИЕ 1.3. Кривая $\{\gamma(s), s \in \mathbb{R}\} \subset E$ называется полной траекторией процесса $\{U(t, \tau)\}$, если

$$
U(t, \tau) \gamma(\tau)=\gamma(t) \quad \forall t \geqslant \tau, \quad \tau \in \mathbb{R} .
$$

ОПРЕДЕЛЕНИЕ 1.4. ЯДро $\mathscr{K}$ процесса $\{U(t, \tau)\}$ в $E$ состоит из объединения всех ограниченных в $E$ полных траекторий этого процесса:

$$
\mathscr{K}=\left\{\gamma(\cdot): \gamma \text { удовлетворяет (1.3) и }\|\gamma(s)\| \leqslant C_{\gamma} \forall s \in \mathbb{R}\right\} .
$$

Множество $\mathscr{K}(\tau)=\{\gamma(\tau): \gamma \in \mathscr{K}\} \subset E$ называется сечением ядра в момент $\tau \in \mathbb{R}$. 
Teорема 1.1 [2]-[4]. Равномерный аттрактор $\mathscr{A}_{\Sigma}$ семейства процессов $\left\{U_{g}(t, \tau)\right\}, g \in \Sigma$, мозсет быть представлен в виде:

$$
\mathscr{A}_{\Sigma}=\bigcup_{g \in \Sigma} \mathscr{K}_{g}(0) .
$$

Здесь $\mathscr{K}_{g}$ обозначает ядро прочесса $\left\{U_{g}(t, \tau)\right\}$. При этом, $\mathscr{K}_{g}$ не пусто для каждого $g \in \Sigma$.

Рассмотрим пространство $\Sigma$, удовлетворяюшее условиям (I) и (II). Из теоремы Арцела-Асколи вытекает

УТВЕРЖДЕНИЕ 1.1 [17]. Транслячионно-инвариантное множество $\Sigma$ (см. (II)) компактно в $C(\mathbb{R} ; H)$, если и только если

(i) мнохество $\{g(0): g \in \Sigma\}$ компактно в $H$;

(ii) $\Sigma$ равностепенно-непрерывно на $\mathbb{R}$, т.е. существует положительная вещественная функция $\alpha(s) \rightarrow 0, s \rightarrow 0+$, такая, что

$$
\left|g\left(s_{1}\right)-g\left(s_{2}\right)\right| \leqslant \alpha\left(\left|s_{1}-s_{2}\right|\right) \quad \forall s_{1}, s_{2} \in \mathbb{R}, \quad \forall g \in \Sigma .
$$

В частности, найдется константа $C_{5}$ такая, что

$$
\sup _{s \in \mathbb{R}}|g(s)| \leqslant C_{5} \quad \forall g \in \Sigma .
$$

ПРИмеР 1.1. Пусть $g(x, s)=g(s)$ является почти периодической функцией со значениями в $H$. Напомним, что в соответствии с определением Бохнера-Америо [18], [19] это означает, что множество $\{g(s+h): h \in \mathbb{R}\}$ предкомпактно в $C_{b}(\mathbb{R} ; H)$ относительно топологии равномерной сходимости на всей числовой оси $\mathbb{R}$. Замыкание

$$
\overline{\{g(s+h): h \in \mathbb{R}\}}^{C_{b}(\mathbb{R} ; H)}=\mathscr{H}(g)
$$

называется оболочкой $g$ в $C_{b}(\mathbb{R} ; H)$. Пусть теперь $\Sigma=\mathscr{H}\left(g_{0}\right)$, где $g_{0}$ - некоторая почти периодическая функция. Очевидно, множество $\Sigma$ также компактно в $C(\mathbb{R} ; H)$ и свойство (I) выполнено. Условие (II) также имеет место.

ПримеР 1.2. Функция $g_{0}(x, s)=g_{0}(s)$ со значениями в $H$ называется квазипериодической по времени, если

$$
g_{0}(s)=\varphi\left(\alpha_{1} s, \alpha_{2} s, \ldots, \alpha_{k} s\right)=\varphi(\boldsymbol{\alpha} s),
$$

где $\varphi(\boldsymbol{\omega})=\varphi\left(\omega_{1}, \omega_{2}, \ldots, \omega_{k}\right)$ является $2 \pi$-периодической функцией по каждому аргументу $\omega_{j}, j=1, \ldots, k, \varphi(\boldsymbol{\omega}) \in C^{1}\left(\mathbb{T}^{k} ; H\right), \boldsymbol{\omega} \in \mathbb{T}^{k},\left(\alpha_{1}, \alpha_{2}, \ldots, \alpha_{k}\right)=\boldsymbol{\alpha}-$ рационально независимые числа. Легко убедиться в том, что квазипериодическая функция является также почти периодической. Оболочкой квазипериодической функции является (см. [2]) множество

$$
\mathscr{H}\left(g_{0}\right)=\left\{\varphi\left(\boldsymbol{\alpha} s+\omega_{0}\right): \omega_{0} \in \mathbb{T}^{k}\right\} .
$$

Таким образом, оболочкой квазипериодической функции является гладкий образ $k$-мерного тора $\mathbb{T}^{k}$.

Рассмотрим более обший класс функций $g$. 
ОПРЕДЕЛЕНИЕ 1.5. Функция $g(s) \in C(\mathbb{R} ; H)$ называется трансляиионно-компактной в $C(\mathbb{R} ; H)$, если множество $\{g(s+h): h \in \mathbb{R}\}$ предкомпактно в $C(\mathbb{R} ; H)$ относительно локальной топологии равномерной сходимости. Множество

$$
\overline{\{g(s+h): h \in \mathbb{R}\}}^{C(\mathbb{R} ; H)}=\mathscr{H}(g)
$$

называется оболочкой $g$ в $C(\mathbb{R} ; H)$.

Например, пусть $g \in C(\mathbb{R}, H)$ и $g(s) \rightarrow g_{ \pm}, s \rightarrow \pm \infty$, где $g_{+}, g_{-} \in H$ и $g_{+} \neq g_{-}$. Тогда, очевидно, $g$ - трансляционно-компактна в $C(\mathbb{R} ; H)$ и $\mathscr{H}(g)=\{g(s+h)$ : $h \in \mathbb{R}\} \cup\left\{g_{+}, g_{-}\right\}$. Ясно, что $g(s)$ не является почти периодической функцией. Из предложения 1.1 вытекает

СлЕДСТВИЕ 1.1. Функция $g(s) \in C(\mathbb{R} ; H)$ является трансляционно-компактной в $C(\mathbb{R} ; H)$ тогда и только тогда, когда

(i) множсество $\{g(s): s \in \mathbb{R}\}$ предкомпактно в $H$;

(ii) функция $g(t)$ равномерно-непрерьвна на $\mathbb{R}$, т.е. существует функция $\alpha(s) \rightarrow 0, s \rightarrow 0+$, такая, что

$$
\left|g\left(s_{1}\right)-g\left(s_{2}\right)\right| \leqslant \alpha\left(\left|s_{1}-s_{2}\right|\right) \quad \forall s_{1}, s_{2} \in \mathbb{R} \text {. }
$$

Пусть $\Sigma=\mathscr{H}\left(g_{0}\right)$, где $g_{0}$ - трансляционно-компактна в $C(\mathbb{R} ; H)$. Очевидно, что $\Sigma$ удовлетворяет (I) и (II).

Другие примеры множеств $\Sigma$, которые удовлетворяют (I) и (II), будут приведены в $\S 4$.

Дадим определение $\varepsilon$-энтропии и фрактальной размерности компактного множества $Y$ в банаховом пространстве $E: Y \Subset E$. Пусть $N_{\varepsilon}(Y)$ обозначает минимальное число открытых шаров в $E$ радиуса $\varepsilon$, которые покрывают $Y$. Очевидно, $N_{\varepsilon}(Y)<\infty$ при любом $\varepsilon>0$, поскольку $Y-$ компактно в $E$.

ОПРЕДЕЛЕНИЕ 1.6 [5]. Число

$$
\mathbf{H}_{\varepsilon}(Y)=\log _{2}\left(N_{\varepsilon}(Y)\right)
$$

называется колмогоровской $\varepsilon$-энтропией, или короче, $\varepsilon$-энтропией множества $Y$ в $E$.

ОПРЕДЕЛЕНИЕ 1.7. (Верхней) фрактальной размерностью множества $Y$ называется величина

$$
\mathbf{d}_{F}(Y)=\limsup _{\varepsilon \rightarrow 0+} \frac{\mathbf{H}_{\varepsilon}(Y)}{\log _{2}(1 / \varepsilon)}=\limsup _{\varepsilon \rightarrow 0+} \frac{\log _{2}\left(N_{\varepsilon}(Y)\right)}{\log _{2}(1 / \varepsilon)} .
$$

Если $Y=M$ есть $m$-мерное компактное подмногообразие в $E$, то $\mathbf{d}_{F}(M)=m$. $\mathrm{B}$ частности, $\mathbf{d}_{F}\left(\mathbb{T}^{k}\right)=k$. В негладком случае фрактальная размерность не обязательно является целым числом.

Рассмотрим теперь систему реакции-диффузии с квазипериодической внешней силой $g_{0}$. Пусть $\Sigma=\mathscr{H}\left(g_{0}\right)$. Так как множество $\mathscr{H}\left(g_{0}\right)$ является гладким образом 
$k$-мерного тора, то $\mathbf{d}_{F}(\Sigma) \leqslant k\left(Y=C_{b}(\mathbb{R} ; H)\right)$. Как было показано в [2], равномерньй аттрактор $\mathscr{A}_{\mathscr{H}\left(g_{0}\right)}$ имеет конечную фрактальную размерность в $H$ и

$$
\mathbf{d}_{F}\left(\mathscr{A}_{\Sigma}\right) \leqslant k+A_{1} k^{n /(n+2)}+A_{2} .
$$

Константы $A_{1}$ и $A_{2}$ зависят от $\Omega$, а также от констант $C_{1}-C_{5}$. В работах [1] и [2] приведены примеры систем реакции-диффузии с квазипериодическими внешними силами, для которых

$$
k \leqslant \mathbf{d}_{F}\left(\mathscr{A}_{\Sigma}\right) .
$$

Следовательно, размерность равномерных аттракторов может неограниченно расти, когда размерность множества $\Sigma$ увеличивается.

В общем случае, когда $g_{0}$ является почти периодической функцией, фрактальная размерность равномерного аттрактора $\mathscr{A}_{\Sigma}$ может быть бесконечной. Таким образом, становится естественной попытка оценить колмогоровскую $\varepsilon$-энтропию $\mathbf{H}_{\varepsilon}\left(\mathscr{A}_{\Sigma}\right)$ равномерного аттрактора $\mathscr{A}_{\Sigma}$, используя информацию о поведении колмогоровской $\delta$-энтропии множества $\Sigma$.

Далее мы будем изучать колмогоровскую $\varepsilon$-энтропию равномерного аттрактора $\mathscr{A}_{\Sigma}$ системы реакции-диффузии с внешними силами, принадлежащими множеству $\Sigma$, которое удовлетворяет условиям (I), (II).

\section{§ 2. Свойство сжатия}

В этом параграфе будет доказано свойство сжатия для траекторий $u(t) \in \mathscr{A}_{\Sigma}$, где $\mathscr{A}_{\Sigma}$ - равномерный аттрактор системы реакции-диффузии $(0.1),(0.2)$. Мы будем использовать метод, разработанный в [7] (см. также [20]). Предполагается, что множество $\Sigma$ удовлетворяет (I) и (II).

Можно доказать, что множество $\mathscr{A}_{\Sigma}$ ограничено в $H$ и в $H_{1}$ :

$$
\left|\mathscr{A}_{\Sigma}\right| \leqslant R_{0}, \quad\left\|\mathscr{A}_{\Sigma}\right\| \leqslant R_{1}
$$

(см., например, [2], [8], [9]), причем,

$$
R_{0} \leqslant \frac{C_{6}}{\nu}, \quad R_{1} \leqslant \frac{C_{7}}{\nu^{3 / 2}}, \quad 0<\nu \leqslant 1,
$$

где константы $C_{6}$ и $C_{7}$ зависят от $\Omega, C_{1}-C_{5}$.

Пусть $g_{1}, g_{2} \in \Sigma$ и $u_{1} \in \mathscr{K}_{g_{1}}, u_{2} \in \mathscr{K}_{g_{2}}$. Очевидно, $u_{1}(t), u_{2}(t) \in \mathscr{A}_{\Sigma}$ для любого $t \in \mathbb{R}$. Функции $u_{1}$ и $u_{2}$ удовлетворяют уравнениям:

$$
\begin{aligned}
& \partial_{t} u_{1}=\nu \Delta u_{1}-f\left(u_{1}\right)+g_{1}(t), \\
& \partial_{t} u_{2}=\nu \Delta u_{2}-f\left(u_{2}\right)+g_{2}(t) .
\end{aligned}
$$

ЗАмечАниЕ 2.1. Теперь мы совершим некоторыепреобразования с этими уравнениями. Отметим, что все формальные выкладки корректны в силу того, что $u_{1}(t), u_{2}(t) \in \mathscr{A}_{\Sigma}$ при всех $t \in \mathbb{R}$, и следовательно, $u_{1}, u_{2} \in C_{\boldsymbol{b}}\left(\mathbb{R} ; H_{1}\right) \cap L_{2}^{\text {loc }}\left(\mathbb{R} ; H_{2}\right)$, $\partial_{t} u_{1}, \partial_{t} u_{2} \in L_{2}^{\text {loc }}(\mathbb{R} ; H)$. Подробное обоснование этой техники преобразований изложено в [9], [16]. 
Обозначим теперь $w=u_{1}-u_{2}, g=g_{1}-g_{2}$. Тогда

$$
\partial_{t} w-\nu \Delta w+f\left(u_{1}\right)-f\left(u_{2}\right)=g .
$$

Предположим, что

$$
\left|g_{1}(\tau)-g_{2}(\tau)\right| \leqslant \delta, \quad t_{0} \leqslant \tau \leqslant t,
$$

где $t_{0}=t-T_{0}$ (величина $T_{0}$ будет точно определена в дальнейшем). Умножая (2.2) на $w$ и интегрируя по $\Omega$, получаем

$$
\frac{1}{2} \frac{d}{d t}|w|^{2}+\nu\|w\|^{2}+\left(f\left(u_{1}\right)-f\left(u_{2}\right), w\right)=(g, w),
$$

$(\cdot, \cdot)$ обозначает скалярное произведение в $\left(L_{2}(\Omega)\right)^{N}$. Из $(1.2)$ следует, что $\left(f\left(v_{1}\right)-\right.$ $\left.f\left(v_{2}\right), v_{1}-v_{2}\right) \geqslant-C_{4}\left|v_{1}-v_{2}\right|^{2}$ для всех $v_{1}, v_{2} \in \mathbb{R}^{N}$. Следовательно, из (2.4) получаем неравенство

$$
\frac{1}{2} \frac{d}{d t}|w|^{2}+\nu\|w\|^{2} \leqslant C_{4}|w|^{2}+(g, w) .
$$

Мы будем изучать величины $\rho(t)=|| w(t) \|^{2} /|w(t)|^{2}\left(\geqslant \lambda_{1}\right)$ и $\xi(t)=|w(t)|$. (Аналогичное исследование проведено в [6].) В силу последнего неравенства имеем

$$
\frac{d}{d t} \xi(t)+\nu \rho(t) \xi(t) \leqslant C_{4} \xi(t)+\delta .
$$

Для функции $\rho$ получаем

$$
\begin{aligned}
\frac{d \rho}{d t} & =\frac{d}{d t} \frac{(\nabla w, \nabla w)}{|w|^{2}}=\frac{2\left(\nabla w^{\prime}, \nabla w\right)}{|w|^{2}}-\frac{2\|w\|^{2}\left(w^{\prime}, w\right)}{|w|^{4}} \\
& =\frac{2}{|w|^{2}}\left(w^{\prime},-\Delta w-\rho w\right)=\frac{2}{|w|^{2}}\left(\nu \Delta w-f\left(u_{1}\right)+f\left(u_{2}\right)+g,-\Delta w-\rho w\right) .
\end{aligned}
$$

Легко проверить, что

$$
(\Delta w,-\Delta w-\rho w)=-|\Delta w+\rho w|^{2} .
$$

В самом деле,

$$
\begin{aligned}
(\Delta w+\rho w, \Delta w+\rho w) & =(\Delta w, \Delta w+\rho w)+\rho(\Delta w, w)+\rho^{2}(w, w) \\
& =(\Delta w, \Delta w+\rho w)-\rho\|w\|^{2}+\rho\|w\|^{2}=(\Delta w, \Delta w+\rho w) .
\end{aligned}
$$

Следовательно, в силу (2.6)

$$
\begin{aligned}
\frac{d \rho}{d t}+\frac{2 \nu}{|w|^{2}}|\Delta w+\rho w|^{2} & =\frac{2}{|w|^{2}}\left(-f\left(u_{1}\right)+f\left(u_{2}\right)+g,-\Delta w-\rho w\right) \\
& \leqslant \frac{2}{|w|^{2}}|g||\Delta w+\rho w|+\frac{2}{|w|^{2}}\left|f\left(u_{1}\right)-f\left(u_{2}\right)\right||\Delta w+\rho w| .
\end{aligned}
$$


Поскольку

$$
f\left(v_{1}\right)-f\left(v_{2}\right)=\left(\int_{0}^{1} f_{v}\left(v_{2}+\theta\left(v_{1}-v_{2}\right)\right) d \theta\right)\left(v_{1}-v_{2}\right)
$$

для всех $v_{1}, v_{2} \in \mathbb{R}^{N}$, используя (1.2) и неравенство Гёльдера, мы получаем

$$
\begin{aligned}
\left|f\left(u_{1}\right)-f\left(u_{2}\right)\right|^{2}= & \int_{\Omega}\left|\left(\int_{0}^{1} f_{v}\left(u_{1}(x)+\theta\left(u_{2}(x)-u_{1}(x)\right)\right) d \theta\right) w(x)\right|^{2} d x \\
\leqslant & C_{8} \int_{\Omega}\left(1+\left|u_{1}(x)\right|^{p-2}+\left|u_{2}(x)\right|^{p-2}\right)^{2}|w(x)|^{2} d x \\
\leqslant & C_{8}\left(\int_{\Omega}\left(1+\left|u_{1}(x)\right|^{p-2}+\left|u_{2}(x)\right|^{p-2}\right)^{n} d x\right)^{2 / n} \\
& \times\left(\int_{\Omega}|w(x)|^{2 n /(n-2)} d x\right)^{(n-2) / n} .
\end{aligned}
$$

Для краткости рассмотрим случай $n \geqslant 3$ (для $n=1,2$ рассуждения аналогичны). Из условия $p \leqslant 2(n-1) /(n-2)$ следует, что $q=(p-2) n \leqslant 2 n /(n-2)$ при $n \geqslant 3$. Следовательно, из вложения $H_{0}^{1}(\Omega) \subset L_{q}(\Omega)$ получаем

$$
\begin{aligned}
\left|f\left(u_{1}\right)-f\left(u_{2}\right)\right|^{2} & \leqslant C_{9}\left(1+\left\|u_{1}\right\|^{(p-2)}+\left\|u_{2}\right\|^{(p-2)}\right)^{2}\|w\|^{2} \\
& \leqslant C_{10}\left(1+R_{1}^{(p-2)}\right)^{2}\|w\|^{2} .
\end{aligned}
$$

Поэтому

$$
\begin{aligned}
\frac{d \rho}{d t} & +\frac{2 \nu}{|w|^{2}}|\Delta w+\rho w|^{2} \\
& \leqslant \frac{2}{|w|^{2}}|g||\Delta w+\rho w|+\frac{2}{|w|^{2}} C_{10}^{1 / 2}\left(1+R_{1}^{(p-2)}\right)\|w\||\Delta w+\rho w| \\
& \leqslant \frac{1}{\nu|w|^{2}}|g|^{2}+\frac{2 \nu}{|w|^{2}}|\Delta w+\rho w|^{2}+\frac{1}{\nu|w|^{2}} C_{10}\left(1+R_{1}^{(p-2)}\right)^{2}\|w\|^{2}
\end{aligned}
$$

и

$$
\frac{d \rho}{d t} \leqslant \frac{1}{\nu|w|^{2}}|g|^{2}+\frac{1}{\nu} C_{10}\left(1+R_{1}^{(p-2)}\right)^{2} \rho .
$$

Мы доказали два неравенства:

$$
\begin{gathered}
\frac{d}{d t} \xi+\nu \rho(t) \xi \leqslant C_{4} \xi+\delta \\
\frac{d \rho}{d t} \leqslant \frac{1}{\nu \xi^{2}} \delta^{2}+\frac{1}{\nu} C_{10}\left(1+R_{1}^{(p-2)}\right)^{2} \rho .
\end{gathered}
$$

Если $\nu \rho(\tau) \geqslant 2 C_{4}\left(\Leftrightarrow \rho(\tau) \geqslant \rho_{0}=2 C_{4} / \nu\right)$ при всех $\tau \in\left[t_{0}, t\right]$, то в силу $(2.8)$

$$
\frac{d \xi}{d t}+\frac{\nu \rho}{2} \xi \leqslant \delta, \quad \rho(\tau) \geqslant \rho_{0} \forall \tau \in\left[t_{0}, t\right]
$$


Если $\xi(\tau) \geqslant \xi_{0}=\delta /\left(\nu \lambda_{1}\right)$ при всех $\tau \in\left[t_{0}, t\right]$, то в силу $(2.9)$

$$
\frac{d \rho}{d t} \leqslant \nu \lambda_{1}^{2}+\alpha \rho, \quad \xi(\tau) \geqslant \xi_{0} \forall \tau \in\left[t_{0}, t\right]
$$

где $\alpha=\nu^{-1} C_{10}\left(1+R_{1}^{(p-2)}\right)^{2}$.

Теперь, используя (2.11), получаем

$$
\begin{aligned}
\rho(t) & \leqslant \rho\left(t_{0}\right) \exp \left(\alpha\left(t-t_{0}\right)\right)+\nu \lambda_{1}^{2} \int_{t_{0}}^{t} \exp (\alpha(t-\tau)) d \tau \\
& \leqslant \rho\left(t_{0}\right) \exp \left(\alpha\left(t-t_{0}\right)\right)+\frac{\nu \lambda_{1}^{2}}{\alpha} \exp \left(\alpha\left(t-t_{0}\right)\right) .
\end{aligned}
$$

Следовательно,

$$
\begin{aligned}
\rho(t) & \leqslant\left(\rho\left(t_{0}\right)+\frac{\nu \lambda_{1}^{2}}{\alpha}\right) \exp \left(\alpha\left(t-t_{0}\right)\right) \\
& =\left(\rho\left(t_{0}\right)+\frac{\nu \lambda_{1}^{2}}{\alpha}\right) \exp \left(\alpha T_{0}\right), \quad T_{0}=t-t_{0} .
\end{aligned}
$$

Из (2.10) вытекает, что

$$
\xi(t) \leqslant \xi\left(t_{0}\right) \exp \left(-\int_{t_{0}}^{t} \frac{\nu \rho(\tau)}{2} d \tau\right)+\delta \int_{t_{0}}^{t} \exp \left(-\int_{\tau}^{t} \frac{\nu \rho(s)}{2} d s\right) d \tau .
$$

Подчеркнем, что (2.12) и (2.13) имеют место при условии, что

$$
\xi(\tau) \geqslant \xi_{0}, \quad \rho(\tau) \geqslant \rho_{0} \quad \forall \tau \in\left[t_{0}, t\right]
$$

С другой стороны, очевидно, $\rho(\tau)=\|w\|^{2} /|w|^{2} \geqslant \lambda_{1}$. Поэтому из (2.13) следует

$$
\begin{aligned}
\xi(t) & \leqslant \xi\left(t_{0}\right) \exp \left(-\frac{\nu \lambda_{1}}{2}\left(t-t_{0}\right)\right)+\delta \int_{t_{0}}^{t} \exp \left(-\frac{\nu \lambda_{1}}{2}(t-\tau)\right) d \tau \\
& =\xi\left(t_{0}\right) \exp \left(-\frac{\nu \lambda_{1}}{2}\left(t-t_{0}\right)\right)+\frac{2 \delta}{\nu \lambda_{1}}\left(1-\exp \left(-\frac{\nu \lambda_{1}}{2}\left(t-t_{0}\right)\right)\right) \\
& =2 \xi_{0}+\exp \left(-\frac{\nu \lambda_{1}}{2}\left(t-t_{0}\right)\right)\left(\xi\left(t_{0}\right)-2 \xi_{0}\right),
\end{aligned}
$$

где $2 \xi_{0}=2 \delta /\left(\nu \lambda_{1}\right)$. А значит,

$$
\xi(t)-2 \xi_{0} \leqslant \exp \left(-\frac{\nu \lambda_{1}}{2}\left(t-t_{0}\right)\right)\left(\xi\left(t_{0}\right)-2 \xi_{0}\right)
$$

В последнем неравенстве, очевидно, $t_{0}$ может быть заменено на любое $\tau \in\left[t_{0}, t\right]$. Таким образом, если $\xi(t) \geqslant 2 \xi_{0}$, то $\xi(\tau) \geqslant 2 \xi_{0}$ для любого $\tau \in\left[t_{0}, t\right]$. И мы имеем $(2.12)$ и (2.13) при $\tau \in\left[t_{0}, t\right]$, если известно, что $\rho(\tau) \geqslant \rho_{0}$ при всех $\tau \in\left[t_{0}, t\right]$. 
В неравенстве (2.12), очевидно, можно заменить $t_{0}$ любым $\tau \in\left[t_{0}, t\right]$, т.е.

$$
\rho(\tau)+\frac{\nu \lambda_{1}^{2}}{\alpha} \geqslant \rho(t) \exp (-\alpha(t-\tau)) \geqslant \rho(t) \exp \left(-\alpha T_{0}\right)
$$

Начиная с этого места, будем предполагать, что

$$
T_{0}=\alpha^{-1}=\nu C_{10}^{-1}\left(1+R_{1}^{(p-2)}\right)^{-2},
$$

т.е. $\rho(\tau)+\nu \lambda_{1}^{2} / \alpha \geqslant \rho(t) e^{-1}, \tau \in\left[t-T_{0}, t\right]$. Наконец, если $e^{-1} \rho(t) \geqslant \rho_{0}+\nu \lambda_{1}^{2} / \alpha$, то, очевидно,

$$
\rho(\tau) \geqslant \rho_{0} \quad \forall \tau \in\left[t-T_{0}, t\right] .
$$

Следовательно, если $\xi(t) \geqslant 2 \xi_{0}$ и $\rho(t) \geqslant e\left(\rho_{0}+\nu \lambda_{1}^{2} / \alpha\right)$, то (2.12) и (2.13) выполнены при всех $\tau \in\left[t-T_{0}, t\right]$. Более того,

$$
\rho(\tau) \geqslant \frac{\rho(t)}{e}-\frac{\nu \lambda_{1}^{2}}{\alpha}=\frac{1}{2 e} \rho(t)+\left(\frac{1}{2 e} \rho(t)-\frac{\nu \lambda_{1}^{2}}{\alpha}\right) .
$$

Предположим теперь, что

$$
\rho(t) \geqslant \rho_{1} \equiv 2 e\left(\rho_{0}+\frac{\nu \lambda_{1}^{2}}{\alpha}\right)
$$

T.e.

$$
\frac{1}{2 e} \rho(t)-\frac{\nu \lambda_{1}^{2}}{\alpha} \geqslant \rho_{0}
$$

Тогда последний член в (2.15) положителен. Из (2.15) и (2.16) вытекает, что

$$
\rho(\tau) \geqslant \frac{1}{2 e} \rho(t) \geqslant \rho_{0}+\frac{\nu \lambda_{1}^{2}}{\alpha} \geqslant \rho_{0} \quad \forall \tau \in\left[t-T_{0}, t\right] .
$$

Используя (2.13) и (2.17), получаем, что

$$
\begin{aligned}
& \xi(t) \leqslant \exp \left(-\frac{\nu}{4 e} \rho(t)\left(t-t_{0}\right)\right) \xi\left(t_{0}\right)+\frac{4 e \delta}{\nu \rho(t)}\left(1-\exp \left(-\frac{\nu}{4 e} \rho(t)\left(t-t_{0}\right)\right)\right) \\
& \xi(t)-\frac{4 e \delta}{\nu \rho(t)} \leqslant \exp \left(-\frac{\nu}{4 e} \rho(t)\left(t-t_{0}\right)\right)\left(\xi\left(t_{0}\right)-\frac{4 e \delta}{\nu \rho(t)}\right) \\
& \leqslant \exp \left(-\frac{\nu}{4 e} \rho(t)\left(t-t_{0}\right)\right) \xi\left(t_{0}\right)
\end{aligned}
$$

Значит, если $\xi(t) \geqslant 8 e \delta /\left(\nu \rho_{1}\right)$ (и конечно, $\rho(t) \geqslant \rho_{1}, \xi(t) \geqslant 2 \xi_{0}$ ), то

$$
\frac{1}{2} \xi(t) \leqslant \xi(t)-\frac{4 e \delta}{\nu \rho(t)}
$$

и в силу (2.18) получаем

$$
\xi(t) \leqslant 2 \exp \left(-\frac{\nu}{4 e} \rho(t)\left(t-t_{0}\right)\right) \xi\left(t_{0}\right) .
$$

Мы доказали следуюшее 
УТВЕРЖДЕНИЕ 2.1. Если

$$
\xi(t) \geqslant \delta \max \left\{\frac{2}{\nu \lambda_{1}} ; \frac{8 e}{\nu \rho_{1}}\right\}=\frac{\delta}{\nu \lambda_{1}} \Gamma_{1}
$$

$u \rho(t) \geqslant \rho_{1}, m o$

$$
\xi(t) \leqslant 2 \exp \left(-\frac{\nu}{4 e} \rho(t) T_{0}\right) \xi\left(t-T_{0}\right)
$$

əде $\rho_{1}=2 e\left(2 C_{4} / \nu+\nu \lambda_{1}^{2} / \alpha\right), T_{0}=\alpha^{-1}, \alpha=C_{10}\left(1+R_{1}^{(p-2)}\right)^{2} \nu^{-1}, R_{1}=C_{7} / \nu^{3 / 2}$, $\Gamma_{1} \equiv \max \left\{2 ; 8 e \lambda_{1} / \rho_{1}\right\}$.

Рассмотрим теперь проекторы $P_{m}$ и $Q_{m}, P_{m} H \oplus Q_{m} H=H, P_{m} h=$ $\sum_{i=1}^{m}\left(h, w_{i}\right) w_{i}$, где $\left\{w_{i}\right\}-$ собственные функции оператора $-\Delta$ с нулевыми граничньми условиями: $-\Delta w_{i}=\lambda_{i} w_{i},\left.w_{i}\right|_{\partial \Omega}=0,0<\lambda_{1} \leqslant \lambda_{2} \leqslant \cdots$.

Если

$$
\left|Q_{m} w\right|^{2} \geqslant\left|P_{m} w\right|^{2}
$$

то, очевидно,

$$
\rho=\frac{\|w\|^{2}}{|w|^{2}}=\frac{\left\|Q_{m} w\right\|^{2}+\left\|P_{m} w\right\|^{2}}{\left|Q_{m} w\right|^{2}+\left|P_{m} w\right|^{2}} \geqslant \frac{\left\|Q_{m} w\right\|^{2}}{2\left|Q_{m} w\right|^{2}} \geqslant \frac{\lambda_{m+1}}{2} .
$$

Из утверждения 2.1 вытекает

Tеорема 2.1 (свойство сжатия). Пусть $\lambda_{m+1} \geqslant 2 \rho_{1} u\left|g_{1}(\tau)-g_{2}(\tau)\right|<\delta n p u$ $\tau \in\left[t-T_{0}, t\right]$. Пусть также $u_{1} \in \mathscr{K}_{g_{1}}, u_{2} \in \mathscr{K}_{g_{2}}$. Если

$$
\left|Q_{m}\left(u_{1}(t)-u_{2}(t)\right)\right|^{2} \geqslant\left|P_{m}\left(u_{1}(t)-u_{2}(t)\right)\right|^{2},
$$

mo

$$
\xi(t)=\left|u_{1}(t)-u_{2}(t)\right|<\max \left\{\frac{\delta}{\nu \lambda_{1}} \Gamma_{1} ; \theta\left|u_{1}\left(t-T_{0}\right)-u_{2}\left(t-T_{0}\right)\right|\right\}
$$

ฉдe

$$
\theta=2 \exp \left(-\frac{\nu \lambda_{m+1}}{8 e} T_{0}\right)
$$

ЗАмечАниЕ 2.2. Отметим асимптотическое поведение параметров $\rho_{1}, T_{0}, \Gamma_{1}$, где $\nu$ мало. Очевидно,

$$
\rho_{1}=O\left(\nu^{-1}\right), \quad T_{0}=O\left(\nu^{(3 p-5)}\right), \quad(3 p-5) \leqslant \frac{n+4}{n-2}, \quad \Gamma_{1}=2 .
$$




\section{§3. Колмогоровская $\varepsilon$-энтропия равномерного аттрактора $\mathscr{A}_{\Sigma}$}

Отметим, что в силу (II)

$$
\Sigma_{l T_{0},(l+1) T_{0}}=\Sigma_{m T_{0},(m+1) T_{0}} \quad \forall l, m \in \mathbb{Z},
$$

где $\Sigma_{a, b}$ обозначает множество функций $g \in \Sigma$, ограниченных на интервал $[a, b]$. Пусть $\mathscr{A}_{\Sigma}=\mathscr{A}$ - равномерный аттрактор семейства процессов, отвечаюших задаче $(0.1),(0.2)$. Пусть

$$
S_{k}(g)=\mathscr{K}_{g ; 0, k T_{0}}
$$

обозначает множество решений $u \in \mathscr{K}_{g}$, ограниченных на интервал $\left[0, k T_{0}\right]$ для каждого $g \in \Sigma$. Целое число $k$ будет определено далее. Пусть также

$$
S_{k}(\Sigma)=\bigcup_{g \in \Sigma} S_{k}(g)
$$

Поскольку множество $\Sigma_{0, k T_{0}}$ компактно в $C\left(\left[0, k T_{0}\right] ; H\right)$ (условие $\left.(\mathrm{I})\right)$, оно может быть покрыто конечным числом шаров $B\left(\varphi_{j}, \delta\right), j=1, \ldots, N, N=N(\delta, k)$ при любом $\delta>0$ :

$$
\Sigma_{0, k T_{0}} \subseteq \bigcup_{j=1}^{N} B\left(\varphi_{j}, \delta\right), \quad B\left(\varphi_{j}, \delta\right) \subset C\left(\left[0, k T_{0}\right] ; H\right) .
$$

Здесь $B(\varphi, \delta)$ - шар в $C\left(\left[0, k T_{0}\right] ; H\right)$, имеюший центр $\varphi$ и радиус $\delta$. Тогда

$$
S_{k}(\Sigma)=\bigcup_{j=1}^{N}\left(\bigcup_{g \in B\left(\varphi_{j}, \delta\right) \cap \Sigma} S_{k}(g)\right) \stackrel{\text { def }}{\equiv} \bigcup_{j=1}^{N} S_{k}^{j} .
$$

Мы будем исследовать множества

$$
\mathscr{A}^{j}\left(t_{i}\right)=\left\{u\left(t_{i}\right): u \in S_{k}^{j}\right\}, \quad t_{i}=i T_{0}, \quad i=0, \ldots, k .
$$

Пусть $u_{1}(t), u_{2}(t) \in S_{k}^{j}$ и они являются решениями $(0.1),(0.2)$ с внешними силами $g_{1}, g_{2} \in \Sigma$, принадлежашими $B\left(\varphi_{j}, \delta\right)$. Пусть $B_{r}\left(u_{0}\right)$ обозначает шар в $H$ с центром в $u_{0}$ и радиусом $r$ такой, что $u_{1}\left(t_{i}\right), u_{2}\left(t_{i}\right) \in B_{r}\left(u_{0}\right)$ для некоторого фиксированного $i=0, \ldots, k-1$. По теореме 2.1 , если $\lambda_{m+1} \geqslant 2 \rho_{1}$, то поскольку $\left|g_{1}(\tau)-g_{2}(\tau)\right| \leqslant 2 \delta, \tau \in\left[t_{i}, t_{i+1}\right]$, или

$$
\left|Q_{m}\left(u\left(t_{i+1}\right)-v\left(t_{i+1}\right)\right)\right|^{2}<\left|P_{m}\left(u\left(t_{i+1}\right)-v\left(t_{i+1}\right)\right)\right|^{2},
$$

или

$$
\left|u\left(t_{i+1}\right)-v\left(t_{i+1}\right)\right| \leqslant \max \left\{\Gamma_{1} \frac{2 \delta}{\lambda_{1} \nu} ; \theta\left|u\left(t_{i}\right)-v\left(t_{i}\right)\right|\right\} \leqslant \max \left\{\Gamma_{1} \frac{2 \delta}{\lambda_{1} \nu} ; 2 \theta r\right\},
$$

где

$$
\theta=2 \exp \left(-\frac{\nu \lambda_{m+1}}{8 e} T_{0}\right), \quad t_{i+1}-t_{i}=T_{0}
$$


Рассмотрим множество

$$
\mathfrak{N}=\mathfrak{N}_{i}^{j}=\left\{u\left(t_{i+1}\right): u \in S_{k}^{j}, u\left(t_{i}\right) \in B_{r}\left(u_{0}\right)\right\}, \quad i=0, \ldots, k-1 .
$$

Выберем максимальное подмножество $\mathfrak{M} \subseteq \mathfrak{N}$, которое удовлетворяет (3.4). В силу леммы Цорна такое множество, очевидно, существует. Тогда

$$
\operatorname{dist}(u, \mathfrak{M}) \leqslant \max \left\{\Gamma_{1} \frac{2 \delta}{\lambda_{1} \nu} ; 2 \theta r\right\} \quad \forall u \in \mathfrak{N} \backslash \mathfrak{M} .
$$

Если $u_{1}, u_{2} \in S_{k}^{j}, u_{1}\left(t_{i}\right), u_{2}\left(t_{i}\right) \in B_{r}\left(u_{0}\right)$, то для $w=u_{1}-u_{2}$ имеем

$$
\begin{gathered}
\frac{1}{2} \frac{d}{d t}|w|^{2}+\nu \rho|w|^{2} \leqslant 2 \delta|w|+C_{4}|w|^{2}, \\
\frac{d}{d t}|w| \leqslant 2 \delta+C_{4}|w| .
\end{gathered}
$$

Интегрируя, получаем

$$
\begin{aligned}
\left|u_{1}\left(t_{i+1}\right)-u_{2}\left(t_{i+1}\right)\right| & \leqslant \exp \left(C_{4} T_{0}\right)\left|u_{1}\left(t_{i}\right)-u_{2}\left(t_{i}\right)\right|+\exp \left(C_{4} T_{0}\right) C_{4}^{-1} 2 \delta \\
& \leqslant 2 r \Gamma_{2}+2 \delta C_{4}^{-1} \Gamma_{2}, \quad \Gamma_{2}=\exp \left(C_{4} T_{0}\right) .
\end{aligned}
$$

Следовательно,

$$
\operatorname{diam} \mathfrak{N} \leqslant 2 r \Gamma_{2}+2 \delta C_{4}^{-1} \Gamma_{2} .
$$

Выберем теперь $m$ достаточно большим (см. (3.19)) и зафиксируем его. В частности, пусть

$$
\theta \leqslant \frac{1}{8}
$$

Предположим, что $\delta$ и $r$ удовлетворяют неравенству

$$
2 \theta r \geqslant \frac{\Gamma_{1} 2 \delta}{\lambda_{1} \nu}
$$

(см. (3.7)). В силу (3.9) и (3.11)

$$
\operatorname{diam} P_{m} \mathfrak{M} \leqslant 2 r \Gamma_{2}+2 \delta C_{4}^{-1} \Gamma_{2} \leqslant 2 r\left(\Gamma_{2}+\lambda_{1} \nu \theta C_{4}^{-1} \Gamma_{1}^{-1} \Gamma_{2}\right) \equiv r \Gamma_{3},
$$

где $\Gamma_{3}=2 \Gamma_{2}\left(1+\lambda_{1} \nu \theta C_{4}^{-1} \Gamma_{1}^{-1}\right)$.

ЛЕмма 3.1. Шар $B_{R}$ в $\mathbb{R}^{n}$ радиуса $R$ можно покрыть с помощью не более $\varkappa_{n}(R+2)^{n}$ шаров $B_{1}$, где $\varkappa_{n} \leqslant 2^{n}$ (см. [21]).

На самом деле, оценка для $\varkappa_{n}$ может быть сушественно улучшена. Известно, например, что

$$
\lim _{n \rightarrow \infty} \frac{\log _{2} \varkappa_{n}}{n}=0
$$

(подробнее см. в [21]). Величина $\varkappa_{n}$ называется наименьщей плотностью покрыmия $\mathbb{R}^{n}$. 
СЛЕДСТВИЕ 3.1. Любое множество $M \subset \mathbb{R}^{n}$ диаметра $D$ можно покрыть не более $(4 D+4)^{n}$ шарами $B_{1}$ радиуса 1 , чентры которых принадлежат $M$.

ДокАЗАтЕльство. В самом деле, множество $M$ принадлежит шару $B_{D}(m)$ при любом $m \in M$. Поэтому по лемме 3.1 оно может быть покрыто $\varkappa_{n}(2 D+2)^{n} \leqslant$ $(4 D+4)^{n}$ шарами $B_{1 / 2}$. Рассмотрим те из них, которые покрьвают $M$. Для каждого такого шара $B_{1 / 2}\left(a_{i}\right)$ найдется некоторая точка $m_{i} \in M \cap B_{1 / 2}\left(a_{i}\right)$. Следовательно, $B_{1 / 2}\left(a_{i}\right) \subseteq B_{1}\left(m_{i}\right)$ и $\left\{B_{1}\left(m_{i}\right)\right\}$ также покрывают $M, m_{i} \in M$ и \# $\left\{B_{1}\left(m_{i}\right)\right\} \leqslant(4 D+4)^{n}$.

Используя следствие 3.1 , заключаем, что множество $P_{m} \mathfrak{M}$ может быть покрыто

$$
q \leqslant\left(\frac{4 r \Gamma_{3}+4 \theta r}{\theta r}\right)^{m}=\left(\frac{4 \Gamma_{3}}{\theta}+4\right)^{m}
$$

шарами радиуса $\theta r$ с центрами в $P_{m} \mathfrak{M}$, например, $B_{\theta r}\left(P_{m} u_{1}\right), \ldots, B_{\theta r}\left(P_{m} u_{q}\right)$, где $P_{m} u_{i} \in P_{m} \mathfrak{M}$.

Рассмотрим шары $B_{4 \theta r}\left(P_{m} u_{1}\right), \ldots, B_{4 \theta r}\left(P_{m} u_{q}\right)$ в $H$. Для любой $v \in \mathfrak{N}$ сушествует $u \in \mathfrak{M}$ (см. (3.7), (3.11)) такая, что $|u-v| \leqslant 2 \theta r$.

Для $P_{m} u$ найдется некоторая $u_{l}, l \in\{1, \ldots, q\}$, такая, что $P_{m} u \in B_{\theta r}\left(P_{m} u_{l}\right)$. Но $u, u_{l} \in \mathfrak{M}$, поэтому

$$
\left|u-u_{l}\right| \leqslant\left|P_{m} u-P_{m} u_{l}\right|+\left|Q_{m} u-Q_{m} u_{l}\right| \leqslant 2\left|P_{m} u-P_{m} u_{l}\right| \leqslant 2 \theta r .
$$

Следовательно, $v \in B_{4 \theta r}\left(u_{l}\right)$. И значит,

$$
\mathfrak{N} \subset \bigcup_{j=1}^{q} B_{4 \theta r}\left(u_{j}\right) \subset \bigcup_{j=1}^{q} B_{r / 2}\left(u_{j}\right),
$$

где $q$ определено в (3.12). При переходе к последнему включению мы воспользовались неравенством (3.10).

В заключение получаем следуюшую лемму.

ЛЕмма 3.2. Пусть $m$ - фиксированное число, которое удовлетворяет (3.10). Тогда множество $\mathfrak{N}$ может быть покрыто $c$ помощью $q \leqslant\left(4 \Gamma_{3} / \theta+4\right)^{m}$ шаров радиуса $r / 2$ в $H$, әде $r$ удовлетворяет (3.11).

Заметим, что

$$
\mathscr{A}=\bigcup_{j=1}^{N} \mathscr{A}^{j}\left(t_{i}\right)
$$

для любого $i=1, \ldots, k$ (см. (1.4), (3.1) и (3.3)). В то же время в силу (2.1), очевидно, $\mathscr{A} \subset B_{R_{0}}(0)$. Применим лемму $3.2 \mathrm{\kappa}$

$$
\mathfrak{N}=\mathfrak{N}_{0}^{j}(1)=\left\{u\left(t_{1}\right): u \in S^{j}, u\left(t_{0}\right) \in B_{R_{0}}(0)\right\}, \quad t_{0}=0, \quad r=R_{0},
$$

получим покрытие $B_{R_{0} / 2}\left(u_{1, j}\right), \ldots, B_{R_{0} / 2}\left(u_{q, j}\right)$. Значит, множество $\mathfrak{N}_{0}^{j}(1)=$ $\mathscr{A}^{j}\left(t_{1}\right)$ может быть покрыто $q$ шарами радиуса $R_{0} / 2$, если $r=R_{0}$ удовлетворяет (3.11). Рассмотрим $q$ множеств $\mathfrak{N}_{1}^{j}(1), \mathfrak{N}_{1}^{j}(2), \ldots, \mathfrak{N}_{1}^{j}(q)$, где

$$
\mathfrak{N}_{1}^{j}(l)=\left\{u\left(t_{2}\right): u \in S_{k}^{j}, u\left(t_{1}\right) \in B_{R_{0} / 2}\left(u_{l, j}\right)\right\}, \quad l=1, \ldots, q .
$$


Теперь применим лемму 3.2 к каждому из множеств $\mathfrak{N}_{1}^{j}(l)$ и получим $q^{2}$ шаров радиуса $R_{0} / 4$, которые покрывают $\mathscr{A}^{j}\left(t_{2}\right)$. После $k$ таких шагов мы получим семейство $q^{k}$ шаров радиуса $R_{0} / 2^{k}$, которое покрывает множество $\mathscr{A}^{j}\left(t_{k}\right)$ при условии, что

$$
2 \theta \frac{R_{0}}{2^{k-1}} \geqslant \frac{\Gamma_{1} 2 \delta}{\lambda_{1} \nu}
$$

Ho

$$
\mathscr{A}=\bigcup_{j=1}^{N} \mathscr{A}^{j}\left(t_{k}\right), \quad N=N_{\delta}\left(\Sigma_{0, k T_{0}}\right) .
$$

Поэтому доказана

Лемма 3.3. Множество $\mathscr{A}_{\Sigma}$ может быть покрыто с помощью

$$
N_{R_{0} / 2^{k}}\left(\mathscr{A}_{\Sigma}\right) \leqslant N_{\delta}\left(\Sigma_{0, k T_{0}}\right) q^{k}
$$

шаров радиуса $R_{0} / 2^{k}$, әде q и к удовлетворяют (3.12) и (3.15), соответственно.

Пусть $k$ удовлетворяет следуюшему двойному неравенству:

$$
2 \theta \frac{R_{0}}{2^{k}} \geqslant \frac{\Gamma_{1} 2 \delta}{\lambda_{1} \nu}>2 \theta \frac{R_{0}}{2^{k+1}} .
$$

Тогда

$$
\delta>\frac{\theta \lambda_{1} \nu}{\Gamma_{1}} \frac{R_{0}}{2^{k+1}} .
$$

Существуют положительные константы $\bar{c}_{0}, \bar{c}_{1}$, зависящие от формы множества $\Omega$, такие, что

$$
\bar{c}_{0} k^{2 / n} \lambda_{1} \leqslant \lambda_{k} \leqslant \bar{c}_{1} k^{2 / n} \lambda_{1}, \quad k=1,2, \ldots
$$

Следовательно, из (3.6) вытекает, что

$$
2 \exp \left(-\nu T_{0} \lambda_{1} c_{1}(m+1)^{2 / n}\right) \leqslant \theta \leqslant 2 \exp \left(-\nu T_{0} \lambda_{1} c_{0}(m+1)^{2 / n}\right),
$$

где $c_{0}=\bar{c}_{0} /(8 e), c_{1}=\bar{c}_{1} /(8 e)$.

Выберем минимальное целое число $m$, которое удовлетворяет неравенствам

$$
2 \exp \left(-\nu T_{0} \lambda_{1} c_{0}(m+1)^{2 / n}\right) \leqslant \frac{1}{8}, \quad \lambda_{m+1} \geqslant \bar{c}_{0}(m+1)^{2 / n} \lambda_{1} \geqslant 2 \rho_{1},
$$

т.е.

$$
(m+1)^{2 / n} \geqslant \max \left\{\frac{4 \ln 2}{\nu T_{0} \lambda_{1} c_{0}} ; \frac{2 \rho_{1}}{\bar{c}_{0} \lambda_{1}}\right\} \stackrel{\text { def }}{\equiv} D>m^{2 / n} .
$$

Пусть

$$
\frac{R_{0}}{2^{k}} \geqslant \varepsilon>\frac{R_{0}}{2^{k+1}} \Longrightarrow k \leqslant \log _{2} \frac{R_{0}}{\varepsilon} .
$$


Применяя лемму 3.3 с $k+1$ вместо $k$ и используя $(3.18),(3.22),(3.16)$ и (3.17), получаем

$$
\begin{aligned}
N_{\varepsilon}(\mathscr{A}) & \leqslant N_{\frac{R_{0}}{2^{k+1}}}(\mathscr{A}) \leqslant N_{\delta}\left(\Sigma_{0,(k+1) T_{0}}\right) q^{k+1} \\
& \leqslant N_{\frac{\theta \lambda_{1} \nu}{\Gamma_{1}} \frac{R_{0}}{2^{k+1}}}\left(\Sigma_{0,(k+1) T_{0}}\right) q^{k+1} \\
& \leqslant N_{\frac{\theta \lambda_{1} \nu}{2 \Gamma_{1}} \varepsilon}\left(\Sigma_{0,(k+1) T_{0}}\right)\left(\frac{4 \Gamma_{3}}{\theta}+4\right)^{m(k+1)} \\
& \leqslant N_{\frac{\theta \lambda_{1} \nu}{2 \Gamma_{1}} \varepsilon}\left(\Sigma_{0,(k+1) T_{0}}\right)\left(\frac{4 \Gamma_{3}}{\theta}+4\right)^{m \log _{2}\left(2 R_{0} / \varepsilon\right)} .
\end{aligned}
$$

Следовательно, для колмогоровской $\varepsilon$-энтропии справедливо следующее:

$$
\begin{aligned}
\mathbf{H}_{\varepsilon}(\mathscr{A}) & =\log _{2} N_{\varepsilon}(\mathscr{A}) \\
& \leqslant \log _{2}\left(N_{\frac{\theta \lambda_{1} \nu}{2 \Gamma_{1}} \varepsilon}\left(\Sigma_{0,(k+1) T_{0}}\right)\right)+m \log _{2} \frac{2 R_{0}}{\varepsilon} \log _{2}\left(\frac{4 \Gamma_{3}}{\theta}+4\right) \\
& \leqslant \mathbf{H}_{\frac{\theta \lambda_{1} \nu}{2 \Gamma_{1}} \varepsilon}\left(\Sigma_{0, T_{0}} \log _{2}\left(2 R_{0} / \varepsilon\right)\right)+D^{n / 2} \log _{2} \frac{2 R_{0}}{\varepsilon} \log _{2}\left(\frac{4 \Gamma_{3}}{\theta}+4\right) .
\end{aligned}
$$

В результате доказана

Teоpema 3.1. Прu $0<\varepsilon \leqslant R_{0}$

$$
\mathbf{H}_{\varepsilon}(\mathscr{A}) \leqslant \mathbf{H}_{\frac{\theta \lambda_{1} \nu}{2 \Gamma_{1}} \varepsilon}\left(\Sigma_{0, T_{0} \log _{2}\left(2 R_{0} / \varepsilon\right)}\right)+D^{n / 2} \log _{2} \frac{2 R_{0}}{\varepsilon} \log _{2}\left(\frac{4 \Gamma_{3}}{\theta}+4\right),
$$

əдe

$$
D=\max \left\{\frac{4 \ln 2}{\nu T_{0} \lambda_{1} c_{0}} ; \frac{2 \rho_{1}}{\bar{c}_{0} \lambda_{1}}\right\}, \quad \theta=2 \exp \left(-\frac{\nu \lambda_{m+1} T_{0}}{8 e}\right),
$$

а т определено в (3.21).

ЗАмечАниЕ 3.1. Выясним асимптотическое поведение правой части неравенства (3.23), когда $\nu$ мало.

Пусть $\nu=\nu_{0}$ является решением алгебраического уравнения

$$
\frac{4 \ln 2}{\nu T_{0} \lambda_{1} c_{0}}=\frac{2 \rho_{1}}{\bar{c}_{0} \lambda_{1}}
$$

где $T_{0}=T_{0}(\nu), \rho_{1}=\rho_{1}(\nu)$ (см. утверждение 2.1$)$.

Тогда, очевидно, $D=4 \ln \left(2 /\left(\nu T_{0} \lambda_{1} c_{0}\right)\right)$ при $\nu \leqslant \nu_{0}$. Поэтому в силу $(3.19)$ и $(3.21)$

$$
c_{2} \stackrel{\text { def }}{=} 2\left(\frac{1}{16}\right)^{\left(\frac{c_{1}}{c_{0}}\right) 2^{2 / n}} \leqslant 2\left(\frac{1}{16}\right)^{\left(\frac{c_{1}}{c_{0}}\right)\left(\frac{m+1}{m}\right)^{2 / n}} \leqslant \theta
$$

т.е.

$$
c_{2} \leqslant \theta \leqslant \frac{1}{8}
$$


Значит, в силу (3.24) из (3.23) получаем

$$
\mathbf{H}_{\varepsilon}(\mathscr{A}) \leqslant \mathbf{H}_{\frac{c_{2} \lambda_{1} \nu}{2 \Gamma_{1}} \varepsilon}\left(\Sigma_{0, T_{0} \log _{2}\left(2 R_{0} / \varepsilon\right)}\right)+D^{n / 2} \log _{2} \frac{2 R_{0}}{\varepsilon} \log _{2}\left(\frac{4 \Gamma_{3}}{c_{2}}+4\right)
$$

при $0<\nu \leqslant \nu_{0}$.

В соответствии с (2.21) $T_{0}=O\left(\nu^{(3 p-5)}\right)$ и $D=4 \ln 2 /\left(\nu T_{0} \lambda_{1} c_{0}\right)=O\left(\nu^{-(3 p-4)}\right)$ при $0<\nu \leqslant \nu_{0}$. (Мы изучаем случай $2 \leqslant p \leqslant 2(n-1) /(n-2)$.) Для других величин имеем

$$
R_{0}=O\left(\nu^{-1}\right), \quad \Gamma_{1}=2, \quad \Gamma_{3}=2+o(1) .
$$

Следовательно,

$\mathbf{H}_{\varepsilon}(\mathscr{A}) \leqslant \mathbf{H}_{O(\nu) \varepsilon}\left(\Sigma_{0, O\left(\nu^{(3 p-5)}\right) \log _{2}\left(O\left(\nu^{-1}\right) / \varepsilon\right)}\right)+O\left(\frac{1}{\nu^{(3 p-4)}}\right)^{n / 2} \log _{2}\left(\frac{O\left(\nu^{-1}\right)}{\varepsilon}\right)$.

Изучим фрактальную размерность $\mathbf{d}_{F}\left(\mathscr{A}_{\Sigma}\right)$ равномерного аттрактора. Покажем сначала, что величина

$$
\limsup _{\varepsilon_{1} \rightarrow 0+} \frac{\mathbf{H}_{\varepsilon_{1}}\left(\Sigma_{0, \tau \log _{2}\left(K / \varepsilon_{1}\right)}\right)}{\log _{2}\left(1 / \varepsilon_{1}\right)}
$$

не зависит от $K>0$. В самом деле, при $K \geqslant 1$ имеем

$$
\frac{\mathbf{H}_{\varepsilon_{1}}\left(\Sigma_{0, \tau \log _{2}\left(1 / \varepsilon_{1}\right)}\right)}{\log _{2}\left(1 / \varepsilon_{1}\right)} \leqslant \frac{\mathbf{H}_{\varepsilon_{1}}\left(\Sigma_{0, \tau} \log _{2}\left(K / \varepsilon_{1}\right)\right)}{\log _{2}\left(1 / \varepsilon_{1}\right)} .
$$

С другой стороны,

$$
\begin{aligned}
\frac{\mathbf{H}_{\varepsilon_{1}}\left(\Sigma_{0, \tau \log _{2}\left(K / \varepsilon_{1}\right)}\right)}{\log _{2}\left(1 / \varepsilon_{1}\right)} & \leqslant \frac{\mathbf{H}_{\frac{\varepsilon_{1}}{K}}\left(\Sigma_{0, \tau} \log _{2}\left(K / \varepsilon_{1}\right)\right)}{\log _{2}\left(1 / \varepsilon_{1}\right)} \\
& =\frac{\mathbf{H}_{\frac{\varepsilon_{1}}{K}}\left(\Sigma_{0, \tau} \log _{2}\left(K / \varepsilon_{1}\right)\right)}{\log _{2}\left(K / \varepsilon_{1}\right)} \cdot \frac{\log _{2}\left(1 / \varepsilon_{1}\right)+\log _{2} K}{\log _{2}\left(1 / \varepsilon_{1}\right)} .
\end{aligned}
$$

Возьмем $\lim \sup _{\varepsilon_{1} \rightarrow 0+}$ от обеих частей $(3.26)$ и (3.27). Получим

$$
\limsup _{\varepsilon_{1} \rightarrow 0+} \frac{\mathbf{H}_{\varepsilon_{1}}\left(\Sigma_{0, \tau} \log _{2}\left(K / \varepsilon_{1}\right)\right.}{\log _{2}\left(1 / \varepsilon_{1}\right)}=\limsup _{\varepsilon_{1} \rightarrow 0+} \frac{\mathbf{H}_{\varepsilon_{1}}\left(\Sigma_{0, \tau} \log _{2}\left(1 / \varepsilon_{1}\right)\right.}{\log _{2}\left(1 / \varepsilon_{1}\right)} .
$$

Аналогично доказывается случай $K<1$.

Определим следующие величины:

$$
\mathbf{d}_{F}^{\text {loc }}(\Sigma, \tau) \stackrel{\text { def }}{=} \limsup _{\varepsilon_{1} \rightarrow 0+} \frac{\mathbf{H}_{\varepsilon_{1}}\left(\Sigma_{0, \tau} \log _{2}\left(1 / \varepsilon_{1}\right)\right.}{\log _{2}\left(1 / \varepsilon_{1}\right)},
$$

зависящие от $\Sigma$ и положительного параметра $\tau$. 
Если, например, $\Sigma=\mathscr{H}\left(g_{0}\right)$, где $g_{0}$ - гладкая квазипериодическая функция с $k$ независимьми частотами (см. $\S 1)$, то $\mathbf{d}_{F}^{\text {loc }}(\Sigma, \tau)=k$ для любого $\tau$, так как $\mathscr{H}\left(g_{0}\right)$ является липшиц-непрерывным образом $k$-мерного тора $\mathbb{T}^{k}$ и $N_{\varepsilon}\left(\mathbb{T}^{k}\right) \approx C(1 / \varepsilon)^{k}$. Другие примеры будут рассмотрены в дальнейшем.

Если $\mathbf{d}_{F}^{\text {loc }}(\Sigma, \tau)<+\infty$ для некоторого множества $\Sigma$, то будем говорить, что $\Sigma$ имеет локальную фрактальную размерность $\mathbf{d}_{F}^{\text {loc }}(\Sigma, \tau)$ в пространстве $C(\mathbb{R} ; H)$. Положим $\tau=T_{0}$.

СлЕДСТВИЕ 3.2. Предположим, что $\mathbf{d}_{F}^{\mathrm{loc}}\left(\Sigma, T_{0}\right)<+\infty$. Тољда

$$
\mathbf{d}_{F}\left(\mathscr{A}_{\Sigma}\right) \stackrel{\text { def }}{=} \limsup _{\varepsilon \rightarrow 0+} \frac{\mathbf{H}_{\varepsilon}\left(\mathscr{A}_{\Sigma}\right)}{\log _{2}(1 / \varepsilon)} \leqslant \mathbf{d}_{F}^{\text {loc }}\left(\Sigma, T_{0}\right)+D^{n / 2} \log _{2}\left(\frac{4 \Gamma_{3}}{\theta}+4\right) \text {. }
$$

ДокАЗАТЕЛЬСтво. Разделив (3.23) на $\log _{2}(1 / \varepsilon)$ и положив $\varepsilon_{1}=\frac{\theta \lambda_{1} \nu}{2 \Gamma_{1}} \varepsilon$, получим

$$
\begin{aligned}
\mathbf{d}_{F}\left(\mathscr{A}_{\Sigma}\right) & \leqslant \limsup _{\varepsilon_{1} \rightarrow 0+} \frac{\mathbf{H}_{\varepsilon_{1}}\left(\Sigma_{0, T_{0}} \log _{2}\left(\frac{1}{\varepsilon_{1}} \frac{R_{0} \theta \lambda_{1} \nu}{\Gamma_{1}}\right)\right.}{\log _{2} \frac{1}{\varepsilon_{1}}+\log _{2} \frac{2 \Gamma_{1}}{\theta \lambda_{1} \nu}}+D^{n / 2} \log _{2}\left(\frac{4 \Gamma_{3}}{\theta}+4\right) \\
& =\mathbf{d}_{F}^{\mathrm{loc}}\left(\Sigma, T_{0}\right)+D^{n / 2} \log _{2}\left(\frac{4 \Gamma_{3}}{\theta}+4\right) .
\end{aligned}
$$

Здесь мы воспользовались (3.28) с $K=R_{0} \theta \lambda_{1} \nu / \Gamma_{1}$.

Пусть $\gamma(\varepsilon)$ - некоторая положительная монотонная функция такая, что $\gamma(\varepsilon) \rightarrow+\infty, \varepsilon \rightarrow 0+$.

СлЕДСТВИЕ 3.3. Пусть

$$
\frac{\log _{2}(1 / \varepsilon)}{\gamma(\varepsilon)} \rightarrow 0, \quad \varepsilon \rightarrow 0+
$$

и предположим, что для каждого $K>0$

$$
\begin{aligned}
& \limsup _{\varepsilon_{1} \rightarrow 0+} \frac{\mathbf{H}_{\varepsilon_{1}}\left(\Sigma_{0, T_{0} \log _{2}\left(K / \varepsilon_{1}\right)}\right)}{\gamma\left(\varepsilon_{1}\right)} \\
& \quad=\limsup _{\varepsilon_{1} \rightarrow 0+} \frac{\mathbf{H}_{\varepsilon_{1}}\left(\Sigma_{0, T_{0} \log _{2}\left(1 / \varepsilon_{1}\right)}\right)}{\gamma\left(\varepsilon_{1}\right)} \stackrel{\text { def }}{\equiv} \mathbf{d}_{\gamma}^{\operatorname{loc}}\left(\Sigma, T_{0}\right)<+\infty .
\end{aligned}
$$

Тогда

$$
\mathbf{d}_{\gamma}\left(\mathscr{A}_{\Sigma}\right) \stackrel{\text { def }}{=} \limsup _{\varepsilon \rightarrow 0+} \frac{\mathbf{H}_{\varepsilon}\left(\mathscr{A}_{\Sigma}\right)}{\gamma(\varepsilon)} \leqslant \mathbf{d}_{\gamma}^{\text {loc }}\left(\Sigma, T_{0}\right) .
$$

Для доказательства разделим (3.23) на $\gamma(\varepsilon)$. Тогда, переходя к пределу при $\varepsilon \rightarrow 0+$ и используя (3.31), получим (3.32), поскольку второй член

$$
\frac{D^{n / 2} \log _{2}\left(2 R_{0} / \varepsilon\right) \log _{2}\left(4 \Gamma_{3} / \theta+4\right)}{\gamma(\varepsilon)}
$$

стремится к нулю при $\varepsilon \rightarrow 0+$. 
ЗАмечание 3.2. Из неравенства (3.32) следует

$$
N_{\varepsilon}\left(\mathscr{A}_{\Sigma}\right) \leqslant 2^{\left(\mathbf{d}_{\gamma}^{\text {loc }}\left(\Sigma, T_{0}\right)+\rho\right) \gamma(\varepsilon)} \quad \forall \rho>0
$$

для достаточно малых $\varepsilon \leqslant \varepsilon_{0}(\rho)$.

Рассмотрим теперь другие важные характеристики компактного множества $B$ в метрическом пространстве, функииональную размерность $\operatorname{adf}(B)$ и метрический порядок $\mathbf{q}(B)$. По определению (см. [5])

$$
\operatorname{adf}(B)=\limsup _{\varepsilon \rightarrow 0+} \frac{\log _{2} \mathbf{H}_{\varepsilon}(B)}{\log _{2} \log _{2}(1 / \varepsilon)}, \quad \mathbf{q}(B)=\limsup _{\varepsilon \rightarrow 0+} \frac{\log _{2} \mathbf{H}_{\varepsilon}(B)}{\log _{2}(1 / \varepsilon)} .
$$

Очевидно, если $0<\mathbf{d}_{F}^{\text {loc }}(B)<\infty$, то $\mathbf{a d f}(B)=1$ и $\mathbf{q}(B)=0$. Таким образом, величины $\operatorname{adf}(B)$ и $\mathbf{q}(B)$ описывают, в некоторым смысле, множества, имеющие бесконечную фрактальную размерность.

По аналогии с (3.29) и (3.31) определим локальную функциональную размерность $\operatorname{adf}^{\mathrm{loc}}(\Sigma, \tau)$ и локальный метрический порядок $\mathbf{q}^{\mathrm{loc}}(\Sigma, \tau)$ множества $\Sigma_{\text {в }}$ пространстве $C(\mathbb{R} ; H)$ :

$$
\begin{aligned}
\mathbf{a d f}^{\operatorname{loc}}(\Sigma, \tau) & \stackrel{\text { def }}{\equiv} \limsup _{\varepsilon \rightarrow 0+} \frac{\log _{2} \mathbf{H}_{\varepsilon}\left(\Sigma_{0, \tau} \log _{2}(1 / \varepsilon)\right)}{\log _{2} \log _{2}(1 / \varepsilon)}, \\
\mathbf{q}^{\operatorname{loc}}(\Sigma, \tau) & \stackrel{\text { def }}{\equiv} \limsup _{\varepsilon \rightarrow 0+} \frac{\log _{2} \mathbf{H}_{\varepsilon}\left(\Sigma_{0, \tau \log _{2}(1 / \varepsilon)}\right)}{\log _{2}(1 / \varepsilon)} .
\end{aligned}
$$

Заметим, что правые части равенств (3.33) и (3.34) не меняются, если в них заменить $\Sigma_{0, \tau} \log _{2}(1 / \varepsilon)$ на $\Sigma_{0, \tau \log _{2}(K / \varepsilon)}$ при любом $K>0$. Доказательство аналогично приведенному вьше для величины $\mathbf{d}_{F}^{\text {loc }}(\Sigma, \tau)$.

Предположим, что при $\tau=T_{0}$ правая часть (3.33) больше 1 , а правая часть (3.34) больше 0 . Тогда справедливо

СлЕДСТвИЕ 3.4. Для равномерного аттрактора $\mathscr{A}_{\Sigma}$ имеют место следующие неравенства:

$$
\operatorname{adf}\left(\mathscr{A}_{\Sigma}\right) \leqslant \operatorname{adf}^{\mathrm{loc}}\left(\Sigma, T_{0}\right), \quad \mathbf{q}\left(\mathscr{A}_{\Sigma}\right) \leqslant \mathbf{q}^{\mathrm{loc}}\left(\Sigma, T_{0}\right) .
$$

\section{$\S 4$. Оценки для $\varepsilon$-энтропии и метрического порядка некоторых множеств $\Sigma$}

В этом параграфе будут рассмотрены три класса множеств $\Sigma$, которые удовлетворяют условиям (I) и (II). Будут полученњ оценки для $\varepsilon$-энтропии и для метрического порядка множеств из этих классов.

4.1. Некоторые почти периодические функции. Как известно, решения некоторых линейных краевых задач имеют вид:

$$
v_{0}(x, s)=\sum_{k=1}^{\infty}\left(C_{k}^{0} e^{i \mu_{k} s}+\bar{C}_{k}^{0} e^{-i \mu_{k} s}\right) e_{k}(x), \quad \operatorname{Im} \mu_{k}=0 .
$$


Пусть, например, $e_{k}(x)$ - собственные функции эллиптического оператора

$$
\Delta e_{k}(x)-V(x) e_{k}(x)=-\lambda_{k} e_{k}(x), \quad x \in \Omega \Subset \mathbb{R}^{n},\left.\quad e_{k}\right|_{\partial \Omega}=0,
$$

где $\lambda_{k}>0,\left|e_{k}\right|=1, k=1,2, \ldots$ Тогда (4.1) является решением гиперболического уравнения

$$
\begin{gathered}
\partial_{t}^{2} u=\Delta u-V(x) u,\left.\quad u\right|_{\partial \Omega}=0, \\
\mu_{k}=\sqrt{\lambda_{k}}, \quad k=1,2, \ldots
\end{gathered}
$$

(Мы не приводим здесь условий на функцию $V(x)$.) Предполагается, что числа $\left\{\mu_{k}\right\}_{k \in \mathbb{N}}$ рационально независимы. Теперь если

$$
\sum_{k=1}^{\infty}\left|C_{k}^{0}\right|^{2}<+\infty
$$

то (4.1) является почти периодической функцией со значениями в $H=L_{2}(\Omega)$. Предположим, что для некоторого числа $\alpha>0$

$$
\sum_{k=1}^{\infty}|k|^{2 \alpha}\left|C_{k}^{0}\right|^{2}=M^{2}
$$

Отметим, что

$$
\mu_{k}=a_{k}|k|^{1 / n}, \quad 0<a \leqslant a_{k} \leqslant a^{\prime}, \quad k \in \mathbb{N} .
$$

Рассмотрим шкалу пространств $H_{\beta}$, соответствуюшую оператору $\{\Delta e-V(x) e$, $\left.\left.e\right|_{\partial \Omega}=0\right\}, H=H^{0}$. Положим $\beta=n \alpha$. Пусть $v=\Sigma_{k=1}^{\infty} A_{k} e_{k}-$ любая функция из $H_{\beta}$. Очевидно, что следующая норма

$$
\|v\|_{\beta}^{2}=\sum_{k=1}^{\infty} k^{2 \alpha}\left|A_{k}\right|^{2}=\sum_{k=1}^{\infty}\left(k^{1 / n}\right)^{2 n \alpha}\left|A_{k}\right|^{2}=\sum_{k=1}^{\infty}\left(k^{1 / n}\right)^{2 \beta}\left|A_{k}\right|^{2}
$$

эквивалентна норме

$$
\|v\|_{H^{\beta}}^{2}=\sum_{k=1}^{\infty} \mu_{k}^{2 \beta}\left|A_{k}\right|^{2},
$$

поскольку $k^{\alpha}=\left(k^{1 / n}\right)^{n \alpha}=O\left(\mu_{k}^{\beta}\right)$. Ниже мы будем использовать норму $(4.5)$ в $H_{\beta}$.

Условие (4.4) означает, что

$$
v_{0}(\cdot, t) \in H^{\beta}, \quad t \in \mathbb{R} .
$$

Легко доказать, что оболочка $\mathscr{H}\left(v_{0}\right)=\mathscr{H}_{\alpha}\left(v_{0}\right)$ функции $v_{0}(x, s)=v_{0}(t)$ в $C_{b}\left(\mathbb{R} ; H^{\beta}\right)$ состоит из функций

$$
v(s)=\sum_{k=1}^{\infty}\left(C_{k} e^{i \mu_{k} s}+\bar{C}_{k} e^{-i \mu_{k} s}\right) e_{k}, \quad\left|C_{k}\right|=\left|C_{k}^{0}\right|,
$$


и

$$
\|v(s)\|_{\beta}^{2} \leqslant 4 \sum_{k=1}^{\infty}|k|^{2 \alpha}\left|C_{k}\right|^{2}=4 M^{2} .
$$

Это утверждение непосредственно вытекает из условия рациональной независимости чисел $\left\{\mu_{k}\right\}$.

Оболочка $\mathscr{H}_{\alpha}\left(v_{0}\right)$ компактна в $C_{b}(\mathbb{R} ; H)$. Очевидно, для каждой функции $v(s) \in$ $\mathscr{H}\left(v_{0}\right)$ множество соответствуюших коэф фициентов $\left\{C_{k}\right\}_{k \in \mathbb{N}}$ принадлежит эллипсоиду $E_{\alpha}(M)$ :

$$
\sum_{k=1}^{\infty} k^{2 \alpha}\left|C_{k}\right|^{2}=M^{2}
$$

в пространстве $l_{2}=\left\{\left\{C_{k}\right\}_{k \in \mathbb{N}}:\left\|\left\{C_{k}\right\}\right\|_{l_{2}}^{2}=\sum_{k=1}^{\infty}\left|C_{k}\right|^{2}<\infty\right\}$. Вычислим расстояние между любыми двумя функциями $v_{1}(s), v_{2}(s) \in \mathscr{H}_{\alpha}\left(v_{0}\right)$ в $C_{b}(\mathbb{R} ; H)$ :

$$
\left\|v_{1}-v_{2}\right\|_{C_{b}(\mathbb{R} ; H)}^{2}=\sup _{s \in \mathbb{R}}\left\|v_{1}(s)-v_{2}(s)\right\|_{H}^{2} \leqslant 4 \sum_{k=1}^{\infty}\left|C_{k}^{1}-C_{k}^{2}\right|^{2} .
$$

Таким образом,

$$
\mathbf{H}_{\varepsilon}\left(\mathscr{H}_{\alpha}\left(v_{0}\right)\right) \leqslant \mathbf{H}_{\varepsilon / 2}\left(E_{\alpha}(M)\right) \text {. }
$$

Оценим сверху $\varepsilon$-энтропию $\mathbf{H}_{\varepsilon}\left(E_{\alpha}(M), l_{2}\right)=\mathbf{H}_{\varepsilon}\left(E_{\alpha}(M)\right)$ множества $E_{\alpha}(M)$, измеренную в пространстве $l_{2}$. В работе [5] доказано, что $\varepsilon$-энтропия $\mathbf{H}_{\varepsilon}\left(E_{\alpha}(M)\right)$ удовлетворяет неравенству

$$
\mathbf{H}_{\varepsilon}\left(E_{\alpha}(M)\right) \leqslant c\left(\frac{M}{\varepsilon}\right)^{1 / \alpha},
$$

где $c$ зависит от $\alpha$ и $\gamma$ и не зависит от $\varepsilon$. Поскольку множество $\mathscr{H}_{\alpha}\left(v_{0}\right) \Subset C_{b}(\mathbb{R} ; H)$ удовлетворяет (4.8), то из (4.9) следует

TeOpema 4.1. Для $\varepsilon$-әнтропии $\mathbf{H}_{\varepsilon}\left(\mathscr{H}_{\alpha}\left(v_{0}\right)\right)=\mathbf{H}_{\varepsilon}\left(\mathscr{H}_{\alpha}\left(v_{0}\right), C_{b}(\mathbb{R} ; H)\right)$ множсества $\mathscr{H}_{\alpha}\left(v_{0}\right)$, измеренной в $C_{b}(\mathbb{R} ; H)$, справедлива следующая оценка свер$x y$ :

$$
\mathbf{H}_{\varepsilon}\left(\mathscr{H}_{\alpha}\left(v_{0}\right)\right) \leqslant \mathbf{H}_{\varepsilon / 2}\left(E_{\alpha}(M)\right) \leqslant c\left(\frac{2 M}{\varepsilon}\right)^{1 / \alpha}
$$

СЛЕДСТВИЕ 4.1. Для метрического порядка множества $\mathscr{H}_{\alpha}\left(v_{0}\right)$ в $C_{b}(\mathbb{R} ; H)$ получаем

$$
\mathbf{q}\left(\mathscr{H}_{\alpha}\left(v_{0}\right)\right)=\limsup _{\varepsilon \rightarrow 0+} \frac{\log _{2} \mathbf{H}_{\varepsilon}\left(\mathscr{H}\left(v_{0}\right)\right)}{\log _{2}(1 / \varepsilon)} \leqslant \frac{1}{\alpha}
$$

ЗАмечАнИЕ 4.1. На самом деле, в (4.10) имеет место равенство, так как $\mathbf{H}_{\varepsilon}\left(E_{\alpha}(M)\right)$ удовлетворяет аналогичным (4.9) нижним оценкам (подробнее см. в $[5])$.

Теперь, для некоторого заданного вектора $a \in \mathbb{R}^{N}$ рассмотрим множество $\Sigma=a \cdot \mathscr{H}_{\alpha}\left(v_{0}\right) \subset C_{b}(\mathbb{R} ; H)$. Множество $\Sigma$ удовлетворяет (I) и (II). Пусть $\mathscr{A}_{\Sigma}-$ равномерный аттрактор системы реакции-диффузии с таким множеством $\Sigma$. Воспользовавшись теоремой 3.1 , получим оценку для $\mathbf{H}_{\varepsilon}\left(\mathscr{A}_{\Sigma}\right)$. 
СлЕДСТвиЕ 4.2. Справедливо следующее:

$$
\mathbf{H}_{\varepsilon}\left(\mathscr{A}_{\Sigma}\right) \leqslant \mathbf{H}_{\mu \varepsilon}(\Sigma)+\log _{2} \frac{2 R_{0}}{\varepsilon} \log _{2} q \leqslant c\left(\frac{2 M}{\mu \varepsilon}\right)^{1 / \alpha}+\log _{2} \frac{2 R_{0}}{\varepsilon} \log _{2} q
$$

əдe

$$
\mu=\frac{\theta \lambda_{1} \nu}{2 \Gamma_{1}}, \quad q=\left(\frac{4 \Gamma_{3}}{\theta}+4\right)^{D^{n / 2}} .
$$

Мы воспользовались очевидным неравенством

$$
\mathbf{H}_{\mu \varepsilon}\left(\Sigma_{0, \tau}\right) \leqslant \mathbf{H}_{\mu \varepsilon}(\Sigma) .
$$

Используя следствие 4.1 , в силу (4.10) и следствия 3.4 получаем

СлЕДСТВИЕ 4.3. Справедлива оченка

$$
\mathbf{q}\left(\mathscr{A}_{\Sigma}\right) \leqslant \mathbf{q}(\Sigma) \leqslant \frac{1}{\alpha}
$$

ЗАмечание 4.2. Аналогичные результаты, очевидно, имеют место для множества $\Sigma$, состоящего из функций

$$
g(x, s)=\sum_{j=1}^{M} g_{j}(s) a_{j},
$$

где $g_{j} \in \mathscr{H}_{\alpha}\left(v_{j}\right), a_{j} \in \mathbb{R}^{N}, j=1, \ldots, M$. Аналогичньм образом рассматриваются бесконечные суммы в (4.11).

4.2. Класс функций из теории информации. В теории информации (см. [5]) встречаются классы функций $g(s),-\infty<s<+\infty$ (сигналов), имеющих ограниченный спектр. Последнее означает, что преобразование Фурье $\widehat{g}(\lambda)=F_{s \rightarrow \lambda} g(s)$ этих функций имеет компактньй носитель, например, $\operatorname{supp} \widehat{g}(\lambda) \subset[-\sigma, \sigma]$. Отметим, что функции вида

$$
g(s)=\sum_{k=-\infty}^{\infty} C_{k} e^{i \lambda_{k} s}, \quad C_{-k}=\bar{C}_{k}, \quad \lambda_{k}=-\lambda_{-k},
$$

где

$$
\left|\lambda_{k}\right| \leqslant \sigma,
$$

удовлетворяют этому условию. Предполагается также, что

$$
\sum_{k}\left|C_{k}\right|=C<+\infty .
$$

Сразу отметим, что подобная функция $g(s)$ является почти периодической и ее можно продолжить на комплексную плоскость $\mathbb{C}=\{z\}=\{s+i \tau\}$. Соответствуюшая функция $g(z)$ является целой функцией и

$$
|g(s+i \tau)|=|g(z)| \leqslant C e^{\sigma|\operatorname{Im} z|}, \quad z=s+i \tau,
$$

где $\sigma=\sup _{k}\left\{\left|\lambda_{k}\right|\right\}$. 
Следуя Тихомирову (см. [5]), рассмотрим класс $B_{\sigma}(C) \equiv B$ всех целых функций $g(z)$, которые удовлетворяют (4.14). Здесь $C>0$ и $\sigma>0$ - фиксированные числа. Из теоремы Пэли-Винера следует, что преобразование Фурье $\widehat{g}(\lambda)=F_{s \rightarrow \lambda} g(s)$ функции $g(s) \in B_{\sigma}(C)$ имеет компактный носитель, т.е. $g(s)$ имеет ограниченньй спектр. Отметим, что множество $B$ является трансляционно-инвариантным и компактным в $C(\mathbb{R} ; \mathbb{R})$, т.е. оно удовлетворяет (I) и (II).

Обозначим через $B_{[0, T]}=B_{\sigma}(C)_{[0, T]}$ ограничение функций из $B_{\sigma}(C)$ на интервал $[0, T], T>0$. Оценка для $\varepsilon$-энтропии $B_{[0, T]}$ в $C([0, T])$ получена Тихомировым в работе [5]. Он доказал, что минимальное число $N_{\varepsilon}\left(B_{[0, T]}\right)$ шаров в $C([0, T])$, необходимое для покрытия $B_{[0, T]}$, удовлетворяет неравенству

$$
N_{\varepsilon}\left(B_{[0, T]}\right)=N_{\varepsilon}(T) \leqslant\left(2\left[\frac{C^{\prime} C}{\varepsilon}\right]+1\right)^{4[T(\sigma+\alpha) / \pi]+4\left[C^{\prime \prime} C / \varepsilon\right]+6},
$$

где $\alpha$ - некоторая фиксированная постоянная, константы $C^{\prime}$ и $C^{\prime \prime}$ зависят от $\alpha$ и не зависят от $T$ и от $\varepsilon$ (здесь [b] обозначает целую часть $b$ ). Из оценки (4.15) следует, что

$\mathbf{H}_{\varepsilon}\left(B_{[0, T]}\right)=\log _{2} N_{\varepsilon}(T) \leqslant\left(\log _{2} \frac{1}{\varepsilon}+\log _{2}\left(2 C^{\prime} C+1\right)\right) \cdot\left(4 \frac{T(\sigma+\alpha)}{\pi}+\frac{4 C^{\prime \prime} C}{\varepsilon}+6\right)$,

где $\varepsilon<1$. Отсюда для $T=\tau \log _{2}(1 / \varepsilon)$ получаем

УТВеРЖДЕнИЕ 4.1. Справедливы следующие оценки:

$$
\begin{gathered}
\mathbf{H}_{\varepsilon}\left(B_{\left[0, \tau \log _{2}(1 / \varepsilon)\right]}\right) \leqslant C_{1} \frac{1}{\varepsilon} \log _{2} \frac{1}{\varepsilon}, \\
\mathbf{q}^{\mathrm{loc}}(B, \tau)=\limsup _{\varepsilon \rightarrow 0+} \frac{\log _{2} H_{\varepsilon}\left(B_{0, \tau \log _{2}(1 / \varepsilon)}\right)}{\log _{2}(1 / \varepsilon)} \leqslant 1 .
\end{gathered}
$$

Теперь для заданной вектор-функции $a(x) \in H=\left(L_{2}(\Omega)\right)^{N}$ рассмотрим множество $\Sigma=a(x) \cdot B \subset C_{b}(\mathbb{R} ; H)$. Множество $\Sigma$ удовлетворяет (I) и (II). Пусть $\mathscr{A}_{\Sigma}$ обозначает равномерный аттрактор системы реакции-диффузии с таким множеством $\Sigma$. Используя теорему 3.1 и следствие 3.4 , в силу (4.16) получаем

СлЕдСтвиЕ 4.4. Справедливо следующее:

$$
\begin{aligned}
\mathbf{H}_{\varepsilon}\left(\mathscr{A}_{\Sigma}\right) & \leqslant \mathbf{H}_{\mu \varepsilon}\left(\Sigma_{0, T_{0}} \log _{2}\left(2 R_{0} / \varepsilon\right)\right)+\log _{2} \frac{2 R_{0}}{\varepsilon} \log _{2} q \\
& \leqslant C_{1}^{\prime} \frac{1}{\varepsilon} \log _{2} \frac{1}{\varepsilon}+\log _{2} \frac{2 R_{0}}{\varepsilon} \log _{2} q,
\end{aligned}
$$

əде

$$
\mu=\frac{\theta \lambda_{1} \nu}{2 \Gamma_{1}}, \quad q=\left(\frac{4 \Gamma_{3}}{\theta}+4\right)^{D^{n / 2}} .
$$

СлЕДСТВИЕ 4.5. Справедлива оченка

$$
\mathbf{q}\left(\mathscr{A}_{\Sigma}\right) \leqslant \mathbf{q}(\Sigma) \leqslant 1
$$


ЗАмЕчАниЕ 4.3. Очевидно, аналогичные результаты получаются для множества $\Sigma$ функций

$$
g(x, s)=\sum_{j=1}^{M} g_{j}(s) a_{i}(x),
$$

где $g_{j} \in B_{\sigma_{j}}\left(C_{j}\right), a_{j} \in H, j=1, \ldots, M$. Аналогично рассматриваются бесконечные суммы в (4.17).

4.3. Трансляционно-ограниченные функции в пространстве Соболева. Обозначим через $W^{1}$ пространство функций $g \in L_{2}^{\text {loc }}\left(\mathbb{R} ; H_{1}\right), \partial_{t} g \in L_{2}^{\text {loc }}(\mathbb{R} ; H)$, имеющих конечную норму

$$
\|g\|_{W^{1}}^{2}=\sup _{h \in \mathbb{R}} \int_{0}^{1}\left(\|g(s+h)\|^{2}+\left|\partial_{t} g(s+h)\right|^{2}\right) d s<+\infty
$$

Рассмотрим

$$
\Sigma=\Sigma^{M}=\left\{g \in W^{1}:\|g\|_{W^{1}} \leqslant M\right\} .
$$

Если $g \in \Sigma_{M}$, то, очевидно, $\|T(h) g\|_{W^{1}}=\|g\|_{W^{1}}$ и $T(h) g(s)=g(s+h) \in \Sigma_{M}$ для всех $h \in \mathbb{R}$, т.е. (II) выполнено. Проверим условие (I). Положим

$$
W_{t_{1}, t_{2}}^{1}=\left\{g: g \in L_{2}\left(t_{1}, t_{2} ; H_{1}\right), \partial_{t} g \in L_{2}\left(t_{1}, t_{2} ; H\right)\right\}
$$

Из теоремы вложения Соболева вытекает, что $W_{t_{1}, t_{2}}^{1} \Subset C\left(\left[t_{1}, t_{2}\right] ; H\right)$. Пусть дана последовательность $\left\{g_{n}\right\} \subset \Sigma_{M}$, тогда она предкомпактна в $C\left(\left[t_{1}, t_{2}\right] ; H\right)$ для каждого $\left[t_{1}, t_{2}\right] \subset \mathbb{R}$. С помощью метода диагонализации легко выделить подпоследовательность $\left\{g_{n_{k}}\right\}$ такую, что $\left\{g_{n_{k}}(t)\right\}$ сходится к $g(t)$ в $C\left(\left[t_{1}, t_{2}\right] ; H\right)$ при $n_{k} \rightarrow \infty$ для любого $\left[t_{1}, t_{2}\right] \subset \mathbb{R}$ и, значит, $g_{n_{k}}(t) \rightarrow g(t), n_{k} \rightarrow \infty$, в $C(\mathbb{R} ; H)$. С другой стороны, при любом фиксированном $h \in \mathbb{R}$ можно предполагать, что $g_{n_{k}}(t) \rightarrow g(t)$, $n_{k} \rightarrow \infty$, слабо в $W_{h, h+1}^{1}=H^{1}(\Omega \times[h, h+1])$. Но $\left\|g_{n_{k}}\right\|_{W_{h, h+1}^{1}} \leqslant M$. Следовательно, переходя к пределу, $\|g\|_{W_{h, h+1}^{1}} \leqslant M$ для любого $h \in \mathbb{R}$, т.е. $\|g\|_{W^{1}} \leqslant M$ и $g \in \Sigma_{M}$. Мы доказали, что $\Sigma_{M}$ компактно в $C(\mathbb{R} ; H)$.

ЗАмЕчАнИЕ 4.4. Аналогичньм образом можно определить пространство $W^{\alpha}$, $\frac{1}{2}<\alpha<1$, функций $\varphi$ таких, что $\varphi(s+h) \in L_{2}(0,1 ; H) \cap H^{\alpha}(\Omega \times[0,1])$ при любом $h \in \mathbb{R}$ с конечно нормой

$$
\|\varphi\|_{W^{\alpha}}=\sup _{h \in \mathbb{R}}\|\varphi(\cdot+h)\|_{H^{\alpha}(\Omega \times[0,1])} .
$$

Для множества $\Sigma=\Sigma_{M}^{\alpha}=\left\{\varphi \in W^{\alpha}:\|\varphi\|_{W^{\alpha}} \leqslant M\right\}$ выполнены условия (I) и (II). При доказательстве этого утверждения используется компактность вложения $H^{\alpha}(\Omega \times[0,1]) \Subset C([0,1] ; H)$.

Для простоты изложения мы рассмотрим случай $\Omega=(0,1)^{n}$. Очевидно, множество $\Sigma=\Sigma^{M}$ удовлетворяет условиям теоремы 3.1 (см. $\S 3$ ). Следовательно,

$$
N_{\varepsilon}\left(\mathscr{A}_{\Sigma}, H\right) \leqslant N_{\mu \varepsilon}\left(\Sigma_{0,(k+1) T_{0}}, C\left(\left[0,(k+1) T_{0}\right] ; H\right)\right) q^{k+1},
$$


где $\mathscr{A}_{\Sigma}$ - равномерный аттрактор семейства процессов $\left\{U_{g}(t, \tau): g \in \Sigma\right\}$, отвечающего системе реакции-диффузии,

$$
\mu=\frac{\theta \lambda_{1} \nu}{2 \Gamma_{1}}, \quad k=\log _{2} \frac{R_{0}}{\varepsilon}, \quad q=\left(\frac{4 \Gamma_{3}}{\theta}+4\right)^{D^{n / 2}} .
$$

Здесь $N_{\varepsilon}\left(\mathscr{A}_{\Sigma}, H\right)$ обозначает наименьшее число $\varepsilon$-шаров в метрике $H$, покрываюших $\mathscr{A}_{\Sigma}$, и $N_{\mu \varepsilon}\left(\Sigma_{0,(k+1) T_{0}}, C\left(\left[0,(k+1) T_{0}\right] ; H\right)\right)$ - минимальное число $\mu \varepsilon$-шаров в метрике $C\left(\left[0,(k+1) T_{0}\right] ; H\right)$, покрывающих $\Sigma_{0,(k+1) T_{0}}$.

Из (4.19) вытекает, что

$$
\|g\|_{W_{2}^{1}\left(Q_{0,(k+1) T_{0}}\right)} \leqslant M\left((k+1) T_{0}+1\right) \quad \forall g \in \Sigma_{0,(k+1) T_{0}}
$$

где $Q_{0,(k+1) T_{0}}=\left[0,(k+1) T_{0}\right] \times \Omega$. Следовательно,

$$
\begin{aligned}
& N_{\mu \varepsilon}\left(\Sigma_{0,(k+1) T_{0}}, C\left(\left[0,(k+1) T_{0}\right] ; H\right)\right) \\
& \quad \leqslant N_{\mu \varepsilon}\left(\left\{\|g\|_{W_{2}^{1}\left(Q_{0,(k+1) T_{0}}\right)} \leqslant M\left((k+1) T_{0}+1\right)\right\}, C\left(\left[0,(k+1) T_{0}\right] ; H\right)\right) .
\end{aligned}
$$

Пусть число $\varepsilon_{1}>0$ удовлетворяет неравенствам

$$
\mu \varepsilon>\varepsilon_{1}, \quad(k+1) \leqslant \log _{2} \frac{1}{\varepsilon_{1}} .
$$

Тогда

$$
Q_{0,(k+1) T_{0}} \subset Q_{0, \log _{2}\left(1 / \varepsilon_{1}\right) T_{0}}
$$

Следовательно, правая часть (4.23) не превосходит

$$
N_{\varepsilon_{1}}\left(\left\{\|g\|_{W_{2}^{1}\left(Q_{0, T_{0} \log _{2}\left(1 / \varepsilon_{1}\right)}\right)} \leqslant M\left(T_{0} \log _{2} \frac{1}{\varepsilon_{1}}+1\right)\right\}, C\left(\left[0, T_{0} \log _{2} \frac{1}{\varepsilon_{1}}\right] ; H\right)\right)
$$

Обозначим

$$
\left\{g:\|g\|_{W_{2}^{1}\left(Q_{0, T_{0} \log _{2}\left(1 / \varepsilon_{1}\right)}\right)} \leqslant M\left(T_{0} \log _{2} \frac{1}{\varepsilon_{1}}+1\right)\right\}=G_{M, T_{0} \log _{2}\left(1 / \varepsilon_{1}\right)} .
$$

Сделаем замену переменной

$$
t \mapsto \tau=\frac{t}{T_{0} \log _{2}\left(\frac{1}{\varepsilon_{1}}\right)}: \quad g(t)=\widetilde{g}(\tau)=g\left(\tau T_{0} \log _{2} \frac{1}{\varepsilon_{1}}\right)
$$


Новое множество функций $\{\widetilde{g}(\tau)\}$ обозначим $\widetilde{G}$. Имеем

$$
\begin{aligned}
& \left(\int_{0}^{T_{0} \log _{2}\left(1 / \varepsilon_{1}\right)}\left(\|g(t)\|^{2}+\left|\partial_{t} g(t)\right|^{2}\right) d t\right)^{1 / 2} \\
& =\left(\int_{0}^{1}\left(\|\widetilde{g}(\tau)\|^{2}+\left(T_{0} \log _{2} \frac{1}{\varepsilon_{1}}\right)^{-2}\left|\partial_{\tau} \widetilde{g}(\tau)\right|^{2}\right) T_{0} \log _{2} \frac{1}{\varepsilon_{1}} d \tau\right)^{1 / 2} \\
& \leqslant\left(\left(T_{0} \log _{2} \frac{1}{\varepsilon_{1}}\right)^{-1}+T_{0} \log _{2} \frac{1}{\varepsilon_{1}}\right)^{1 / 2} \\
& \times\left(\int_{0}^{1}\left(\|\widetilde{g}(\tau)\|^{2}+\left|\partial_{\tau} \widetilde{g}(\tau)\right|^{2}\right) d \tau\right)^{1 / 2} \\
& \leqslant\left(\left(T_{0} \log _{2} \frac{1}{\varepsilon_{1}}\right)^{-1 / 2}+\left(T_{0} \log _{2} \frac{1}{\varepsilon_{1}}\right)^{1 / 2}\right) \\
& \times\left(\int_{0}^{1}\left(\|\widetilde{g}(\tau)\|^{2}+\left|\partial_{\tau} \widetilde{g}(\tau)\right|^{2}\right) d \tau\right)^{1 / 2}
\end{aligned}
$$

Очевидно,

$$
N_{\varepsilon_{1}}\left(G_{M, T_{0} \log _{2}\left(1 / \varepsilon_{1}\right)}, C\left(\left[0, T_{0} \log _{2} \frac{1}{\varepsilon_{1}}\right] ; H\right)\right)=N_{\varepsilon_{1}}(\widetilde{G}, C([0,1] ; H)) .
$$

Положим

$$
\left\{\widetilde{g}:\|\widetilde{g}(\tau)\|_{W_{2}^{1}\left(Q_{0,1}\right)} \leqslant M\left(T_{0} \log _{2} \frac{1}{\varepsilon_{1}}\right)^{1 / 2}\right\} \equiv \widetilde{G}_{\rho}
$$

где $\rho=M\left(T_{0} \log _{2}\left(1 / \varepsilon_{1}\right)\right)^{1 / 2}$. Из (4.27), (4.28) и (4.30) следует

$$
\widetilde{G} \subset \widetilde{G}_{\rho}
$$

В самом деле, если $\widetilde{g} \in \widetilde{G}_{\rho}$, то $\|\widetilde{g}(\tau)\|_{W_{2}^{1}\left(Q_{0,1}\right)} \leqslant M\left(T_{0} \log _{2}\left(1 / \varepsilon_{1}\right)\right)^{1 / 2}$, и в силу (4.28)

$$
\begin{aligned}
& \left(\int_{0}^{T_{0} \log _{2}\left(1 / \varepsilon_{1}\right)}\left(\|g(t)\|^{2}+\left|\partial_{t} g(t)\right|^{2}\right) d t\right)^{1 / 2} \\
& \quad \leqslant\left(\left(T_{0} \log _{2} \frac{1}{\varepsilon_{1}}\right)^{-1 / 2}+\left(T_{0} \log _{2} \frac{1}{\varepsilon_{1}}\right)^{1 / 2}\right) M\left(T_{0} \log _{2} \frac{1}{\varepsilon_{1}}\right)^{1 / 2} \\
& \quad=M\left(T_{0} \log _{2} \frac{1}{\varepsilon_{1}}+1\right) .
\end{aligned}
$$

Поэтому $g \in G_{M, T_{0} \log _{2}\left(1 / \varepsilon_{1}\right)}$.

Ко всем функциям $\widetilde{g}(\tau) \in \widetilde{G}_{\rho}$ применим оператор сжатия

$$
\frac{\widetilde{g}(\tau)}{\rho}=\widehat{g}(\tau), \quad \widetilde{g}(\tau) \in \widetilde{G}_{\rho}, \quad \widehat{g}(\tau) \in \widehat{G}^{1},
$$

где

$$
\widehat{G}^{1}=\left\{\widehat{g}(\tau):\|\widehat{g}(\tau)\|_{W_{2}^{1}\left(Q_{0,1}\right)} \leqslant 1\right\}
$$


В соответствии с теоремой Бирмана-Соломяка (см. [22]),

$$
N_{\varepsilon_{1} / \rho}\left(\widehat{G}^{1}, C([0,1] ; H)\right) \leqslant 2^{C_{\omega}\left(\rho / \varepsilon_{1}\right)^{\omega}}, \quad \omega=\frac{n+1}{1-\varrho}, \quad 1>\varrho>\frac{1}{2} .
$$

Действительно, из [22] получаем

$$
N_{\eta}\left(\widehat{G}^{1}, W_{2}^{\varrho}\left(Q_{0,1}\right)\right) \leqslant 2^{C_{\omega}(1 / \eta)^{\omega}} .
$$

По теореме вложения Соболева [23]

$$
\begin{gathered}
W_{2}^{\varrho}\left(Q_{0,1}\right) \subset C([0,1] ; H), \\
\|g\|_{C([0,1] ; H)} \leqslant C_{1 \varrho}\|g\|_{W_{2}^{\varrho}\left(Q_{0,1}\right)}, \quad \varrho>\frac{1}{2} .
\end{gathered}
$$

Теперь (4.34) следует непосредственно из (4.35)-(4.37).

Наконец, делая растяжение $\rho \widehat{g}=g$, получаем из (4.34), (4.31) оценку

$$
\begin{aligned}
N_{\varepsilon_{1}}(\widetilde{G}, C([0,1] ; H)) & \leqslant N_{\varepsilon_{1}}\left(\widetilde{G}_{\rho}, C([0,1] ; H)\right) \\
& =N_{\varepsilon_{1} / \rho}\left(\widehat{G}^{1}, C([0,1] ; H)\right) \leqslant 2^{C_{\omega}\left(\rho / \varepsilon_{1}\right)^{\omega}} .
\end{aligned}
$$

Тогда, в силу (4.29)

$$
N_{\varepsilon_{1}}\left(G_{T_{0} \log _{2}\left(1 / \varepsilon_{1}\right)}, C\left(\left[0, T_{0} \log _{2} \frac{1}{\varepsilon_{1}}\right] ; H\right)\right) \leqslant 2^{C_{\omega}\left(\rho / \varepsilon_{1}\right)^{\omega}} .
$$

Из (4.23), (4.25) и (4.38) заключаем

$$
\begin{aligned}
& N_{\mu \varepsilon}\left(\Sigma_{0,(k+1) T_{0}}, C\left(\left[0,(k+1) T_{0}\right] ; H\right)\right) \\
& \quad \leqslant N_{\varepsilon_{1}}\left(G_{T_{0} \log _{2}\left(1 / \varepsilon_{1}\right)}, C\left(\left[0, T_{0} \log _{2} \frac{1}{\varepsilon_{1}}\right] ; H\right)\right) \leqslant 2^{C_{\omega}\left(\rho / \varepsilon_{1}\right)^{\omega}},
\end{aligned}
$$

где $\varepsilon_{1}$ удовлетворяет (4.24). Имея в виду (4.21), получаем

$$
N_{\varepsilon}\left(\mathscr{A}_{\Sigma}, H\right) \leqslant 2^{C_{\omega}\left(\rho / \varepsilon_{1}\right)^{\omega}} q^{k+1},
$$

где $\omega=(n+1) /(1-\rho), 1>\rho>\frac{1}{2}, \varepsilon_{1}$ удовлетворяет неравенствам $\mu \varepsilon>\varepsilon_{1}$, $k+1 \leqslant \log _{2}\left(1 / \varepsilon_{1}\right)$.

Взяв $\log _{2}$ от обеих частей, получим

$$
\mathbf{H}_{\varepsilon}\left(\mathscr{A}_{\Sigma}, H\right) \leqslant C_{\omega}\left(\frac{\rho}{\varepsilon_{1}}\right)^{\omega}+(k+1) \log _{2} q .
$$

Очевидно, (4.24) имеем место, если $\varepsilon_{1}=\varepsilon \mu_{1}$, где

$$
\mu_{1}=\min \left\{\mu ; \frac{1}{2 R_{0}}\right\}=\lambda_{1} \nu \min \left\{\frac{\theta}{2 \Gamma_{1}} ; \frac{1}{2|\Sigma|}\right\} .
$$

Следовательно,

$$
\begin{aligned}
\mathbf{H}_{\varepsilon}\left(\mathscr{A}_{\Sigma}, H\right) \leqslant & C_{\omega}\left(\frac{M\left(T_{0} \log _{2}\left(1 /\left(\varepsilon \mu_{1}\right)\right)\right)^{1 / 2}}{\varepsilon \mu_{1}}\right)^{(n+1) /(1-\varrho)} \\
& +D^{n / 2} \log _{2} \frac{2 R_{0}}{\varepsilon} \log _{2}\left(\frac{4 \Gamma_{3}}{\theta}+4\right)
\end{aligned}
$$

где мы воспользовались обозначениями

$$
\rho=M\left(T_{0} \log _{2} \frac{1}{\varepsilon \mu_{1}}\right)^{1 / 2}, \quad \omega=\frac{n+1}{1-\varrho}, \varrho>\frac{1}{2} .
$$

Доказана 
ТЕОРема 4.2. Пусть выполнены условия теоремы 3.1 , множсество $\Sigma \subset W^{1}$ $u\|g\|_{W^{1}} \leqslant M$ при любом $g \in \Sigma$. Тогда $\varepsilon$-энтропия $\mathbf{H}_{\varepsilon}\left(\mathscr{A}_{\Sigma}, H\right)$ равномерного аттрактора $\mathscr{A}_{\Sigma}$ удовлетворяет неравенству (4.40).

Рассмотрим метрический порядок $\mathbf{q}^{\text {loc }}\left(\Sigma, T_{0}\right)$ множества $\Sigma$. Из (4.39) следует, что

$$
\mathbf{q}^{\operatorname{loc}}\left(\Sigma, T_{0}\right) \leqslant \frac{n+1}{1-\varrho} \quad \forall \varrho>\frac{1}{2}
$$

т.е. $\mathbf{q}^{\text {loc }}\left(\Sigma, T_{0}\right) \leqslant 2(n+1)$. Используя следствие 3.4 , получаем

СлЕДСТВИЕ 4.7. Справедлива оценка

$$
\mathbf{q}^{\mathrm{loc}}\left(\mathscr{A}_{\Sigma}\right) \leqslant \mathbf{q}^{\mathrm{loc}}\left(\Sigma, T_{0}\right) \leqslant 2(n+1)
$$

\section{Список литературы}

1. Chepyzhov V. V., Vishik M.I. Attractors for non-autonomous evolution equations with almost periodic symbols // C. R. Acad. Sci. Paris. Ser. I Math. 1993. V. 316. P. 357-361.

2. Chepyzhov V. V., Vishik M.I. Attractors of non-autonomous dynamical systems and their dimension // J. Math. Pures Appl. 1994. V. 73. № 3. P. 279-333.

3. Chepyzhov V.V., Vishik M.I. Attractors of non-autonomous evolution equations with translation-compact symbols // Oper. Theory Adv. Appl. 1995. V. 78. P. 49-60.

4. Chepyzhov V. V., Vishik M.I. Non-autonomous evolutionary equations with translation-compact symbols and their attractors // C. R. Acad. Sci. Paris. Ser. I Math. 1995. V. 321. P. 153-158.

5. Колмогоров A.H., Тихомиров B. M. $\varepsilon$-энтропия и $\varepsilon$-емкость множеств в функциональных пространствах // УМН. 1959. Т. 14 (86). № 2. С. 3-86.

6. Chepyzhov V.V., Foias C., Vishik M.I. Uniform attractors for non-autonomous 2D Navier-Stokes equations and estimations of their $\varepsilon$-entropy (to appear).

7. Constantin P., Foias C., Temam R. Attractors representing turbulent flows // Mem. Amer. Math. Soc. 1985. V. 53. № 314.

8. Chepyzhov V.V., Vishik M.I. Trajectory attractors for reaction-diffusion systems // Topol. Methods Nonlinear Anal. 1996. V. 7. № 1. P. 49-76.

9. Бабин А. В., Вишик М. И. Аттракторы эволюционных уравнений. М.: Наука, 1989.

10. Sell G. R. Non-autonomous differential equations and topological dynamics I, II // Trans. Amer. Math. Soc. 1967. V. 127. P. 241-283.

11. Sell G. R. Lectures on topological dynamics and differential equations. Princeton, NJ: Van-Nostrand-Rinhold, 1971.

12. Dafermos C.M. Semi-flows associated with compact and almost uniform processes // Math. Systems Theory. 1974. V. 8. P. 142-149.

13. Dafermos C. M. Almost periodic processes and almost periodic solutions of evolution equations // Proceedings of a University of Florida, International Symposium. New York: Academic Press, 1977. P. 43-57.

14. Hale J. K. Asymptotic behaviour of dissipative systems // Math. Surveys Monographs. V. 25. Providence, RI: Amer. Math. Soc., 1987.

15. Haraux A. Systèmes dynamiques dissipatifs et applications. Paris: Masson, 1991.

16. Temam $R$. Infinite-dimensional dynamical systems in mechanics and physics. Applied Mathematics Series. V. 68. New York: Springer-Verlag, 1988.

17. Chepyzhov V. V., Vishik M.I. Evolution equations and their trajectory attractors // J. Math. Pures Appl. 1997. V. 76. № 10. P. 913-964.

18. Amerio L., Prouse G. Abstract Almost Periodic Functions and Functional Equations. New York: Van Nostrand, 1971. 
19. Левитан Б. М., Жиков В.В. Почти периодические функции и дифференциальные уравнения. М.: Изд-во МГУ, 1978.

20. Eden A., Foias C., Nicolaenco B., Temam R. Exponential Attractors for Dissipative Evolution Equations. Research in Appl. Math. V. 37. New York: John Wiley, 1994.

21. Conway J. H., Sloan N.J.A. Sphere Packing, Latices and Groups. New York: SpringerVerlag, 1988.

22. Бирман М. ШІ., Соломяк М. З. Кусочно-полиномиальные приближения функций классов $W_{p}^{a} / /$ Матем. сб. 1967. Т. 73 (115). № 3. С. 331-355.

23. Соболев С. Л. Некоторые применения функционального анализа в математической физике. М.: Наука, 1988.

Институт проблем передачи информации РАН

E-mail: vishik@ippi.ac.msk.su; chep@ippi.ac.msk.su 